CERN-TH.6610/92

TUM-T31-27/92

ZU-TH $36 / 92$

\title{
RESONANCES IN THE WEAK CHIRAL LAGRANGIAN \#
}

\author{
G. Ecker ${ }^{\star}$ \\ CERN \\ CH-1211 Geneva 23 \\ J. Kambor ${ }^{\natural}$ \\ Physik Department, Technische Universität München \\ D-8046 Garching \\ and \\ D. Wyler \\ Theoretische Physik, Universität Zürich \\ CH-8001 Zürich
}

\begin{abstract}
We consider the weak interaction of the octet of pseudoscalar mesons with meson resonances with spin 0,1 to lowest order in the derivative expansion. We determine the resonance contributions to the $O\left(p^{4}\right)$ weak coupling constants. In general, the resonance contributions imply only relations between these constants. If additional assumptions such as factorization or weak deformation are imposed, a more detailed comparison with experiment becomes possible. In contrast to the strong sector, available data do not suggest that all the weak couplings of the $p^{4}$-Lagrangian are dominated by resonance exchange in the factorization approximation. We also give a treatment of singlet external fields.
\end{abstract}

\# Supported in part by the German Bundesministerium für Forschung und Technologie, contract 06 TM 761 and by Schweizerischer Nationalfonds

* Permanent address: Institut für Theoretische Physik, Universität Wien, Boltzmanngasse 5, A-1090 Wien, Austria

$\diamond$ Address after Oct. 1: Dept. of Physics and Astronomy, Univ. of Massachusetts, Amherst, MA 01003, U.S.A.

CERN-TH.6610/92

August 1992 


\section{Introduction}

The decays of $K$ mesons have long been a very important tool for studying the weak interactions; they have shaped in a crucial way our picture of the flavour structure of the fundamental constituents. However, when we ask detailed quantitative questions about the decays, the strong interactions make an answer difficult $[1,2]$. There do not exist rigorous calculational tools to connect the basic $Q C D$ theory to observables. Lattice gauge simulations are still quite far from producing reliable numbers for decay rates, in particular for non-leptonic decays.

In this situation, one looks for semiphenomenological methods which can identify the leading effects. It is well known that the low energy physics of the light pseudoscalar mesons $(\pi, K, \eta)$ can be described by an effective Lagrangian [3]. It reorganizes $Q C D$ into an expansion in the masses and momenta of the light mesons, the only accessible degrees of freedom at small energies.

The effective theory contains a large number of unknown parameters. In principle, they are calculable in terms of the $Q C D$ coupling constant and the heavy quark masses. Despite several attempts to model the effective parameters (for a recent analysis and a complete list of references, see [4]), they must be determined from experiment at present. If it is sufficient to include only the leading terms in the momentum expansion, this effective description is still predictive and testable.

In the last years, this procedure has been carried out to the next-to-leading order for the strong (starting with the work of Gasser and Leutwyler [5,6]; see also Refs. [7,8]) and non-leptonic weak interactions (Kambor, Missimer and Wyler [9]; Ecker, Pich and de Rafael [10]). While the phenomenological parameters in the strong sector can (essentially) all be determined, many of the weak couplings could not be fixed by experiment. Although the situation will improve, a complete determination remains presumably impossible.

It is therefore very desirable to have a phenomenological model for the couplings. It is well possible that they are dominated by a few contributions from heavier states. If so, the large number of constants can be reduced significantly. Recently it was shown by Ecker, Gasser, Pich and de Rafael [11] and by Donoghue, Ramirez and Valencia [12] that meson resonances (such as the $\rho$ meson) largely saturate the coupling constants of the strong chiral Lagrangian.

In this paper we apply this idea to the non-leptonic weak $\Delta S=1$ interactions. A treatment of vector resonances in the factorization approximation has been presented by Isidori and Pugliese [13] for the case of $K \rightarrow \pi \pi$ and $K \rightarrow \pi \pi \pi$ decays. Here, we give the more general description.

Besides modelling the free parameters, it is also important to know how to supplement the purely pseudoscalar chiral weak Lagrangian by other hadronic states 
such as vector mesons or baryons. Indeed, much of the phenomenological treatment in nuclear and particle physics relies on a special role of the spin-1 mesons [14]. Similarly, the picture of vector (and axial-vector) dominance has been used to describe the weak nucleon-nucleon force. It is therefore only natural to include them in the chiral Lagrangian.

Unlike in the case of the strong interactions, where the couplings of resonances to the (pseudoscalar) mesons are experimentally determined (e.g. from $\varrho \rightarrow \pi \pi$ ), the analogous weak terms are not known; one could only calculate them using a specific model. As an additional difficulty, it turns out that there is a rather large number of weak couplings of pseudoscalar mesons to resonances. (An exception are the weak $\varrho N N$ and $\pi N N$ couplings which are phenomenologically determined [15] and have been calculated in Ref. [16].)

Integrating out the heavy particles yields the resonance contributions to the phenomenological parameters of the chiral Lagrangian. These satisfy several relations, which, unfortunately, cannot be tested with present data. However, because of the plethora of pseudoscalar-resonance couplings, the chiral parameters can be reproduced in principle.

In order to get significant tests, several simplifying phenomenological pictures of the effects of the strong dynamics have been proposed. In this paper we shall concentrate on two, the factorization model $[17,18]$ and the weak deformation model [19]. We show that the two models result, in fact, in the same structure of the weak $p^{4}$-terms. While the weak deformation model also predicts the overall scale factor of the weak couplings, it remains a free parameter in the factorization approach.

When these models are combined with resonance dominance, there are only a few free constants which are further reduced if only vector resonances are considered [13]. For $K \rightarrow 2 \pi, 3 \pi$, these resonances improve the lowest order results because they allow to include the effects of the strong counterterms [13]. However, since they do not generate the genuine weak parameters, which are numerically important [20,21], a more detailed analysis is called for. In particular, the effects of other resonances and the scale dependence of the loops must be investigated and compared with the results of the factorization hypothesis.

For the large class of radiative $K$ decays, only $\mathrm{V}$ and $\mathrm{A}$ resonances contribute. These contributions to the weak coupling constants are known to be important [19, 22 , but a comprehensive quantitative analysis will have to wait for more precise data.

The remainder of the paper is organized as follows. In the next section we present the complete $O\left(p^{4}\right)$ weak Lagrangian of the pseudoscalar mesons [9] in a concise fashion. In Sect. 3 the couplings of the resonances to the light mesons are listed and the contributions to the weak couplings of section 2 are calculated. Then, in part 
4, we describe the factorization and the weak deformation models. The numerical analysis is presented in Sect. 5. Finally, some conclusions are drawn in Sect. 6. The case of external singlet fields is described in the Appendix.

\section{The weak chiral octet Lagrangian to order $p^{4}$}

In Ref. [9], the most general Lagrangian for the $\Delta S=1$ non-leptonic processes up to order $p^{4}$ was given. It turns out that for all practical purposes only a reduced number of terms is needed. This simplifies the treatment considerably. In this section, we will present this convenient new basis for the $p^{4}$-Lagrangian.

As usual, the 8 pseudoscalar mesons are grouped into the unitary matrix

$$
U=e^{i \phi / F}
$$

where $\phi=\sum_{a=1}^{8} \lambda_{a} \phi_{a}$ represents the mesons and $F \simeq F_{\pi}\left(F_{\pi}=93 \mathrm{MeV}\right)$. The matrix field $U$ transforms as

$$
U \rightarrow g_{R} U g_{L}^{\dagger}
$$

under $S U(3)_{L} \times S U(3)_{R}$. We also introduce the unitary matrix u

$$
U=u \cdot u .
$$

The following definitions will be useful $[6,11]$ :

$$
\begin{aligned}
D_{\mu} U & =\partial_{\mu} U-i r_{\mu} U+i U l_{\mu} \\
u_{\mu} & =i u^{\dagger} D_{\mu} U u^{\dagger} \\
\Gamma_{\mu} & =\frac{1}{2}\left\{u^{\dagger}\left(\partial_{\mu}-i r_{\mu}\right) u+u\left(\partial_{\mu}-i l_{\mu}\right) u^{\dagger}\right\} \\
\chi_{ \pm} & =u^{\dagger} \chi u^{\dagger} \pm u \chi^{\dagger} u \\
f_{ \pm \mu \nu} & =u F_{\mu \nu}^{L} u^{\dagger} \pm u^{\dagger} F_{\mu \nu}^{R} u \\
\tilde{f}_{ \pm \mu \nu} & =\epsilon_{\mu \nu \alpha \beta} f_{ \pm}^{\alpha \beta} .
\end{aligned}
$$

Here, $l_{\mu}=v_{\mu}-a_{\mu}, r_{\mu}=v_{\mu}+a_{\mu}$ and $\chi=2 B_{0}(s+i p)$ are matrix-valued external fields with spin $=1$ and 0 , respectively, and $F_{\mu \nu}^{L, R}$ are the corresponding field strength tensors [6]. For instance, an external electromagnetic field takes the form

$$
\begin{aligned}
& l_{\mu}=r_{\mu}=-e Q A_{\mu} \\
& Q=\frac{1}{3} \operatorname{diag}(2,-1,-1) .
\end{aligned}
$$

Up to order $p^{4}$, the CP-conserving weak octet chiral Lagrangian reads

$$
\mathcal{L}_{W}^{8}=\mathcal{L}_{W_{2}}^{8}+\mathcal{L}_{W_{4}}^{8}
$$


where the lowest order term is [23]

$$
\mathcal{L}_{W_{2}}^{8}=c_{2}<\lambda_{6} D_{\mu} U^{\dagger} D^{\mu} U>+c_{5}<\lambda_{6}\left(U^{\dagger} \chi+\chi^{\dagger} U\right)>
$$

and

$$
\mathcal{L}_{W_{4}}^{8}=\frac{c_{2}}{F_{\pi}^{2}} \sum N_{i} W_{i}^{8}
$$

denotes the non-leptonic weak octet Lagrangian of $O\left(p^{4}\right)$.

In (2.7) and below, $\langle\ldots\rangle$ stands for the trace, and $c_{2}, c_{5}$ are constants used in Ref. [9]. It turns out $[24,25,26,9]$ that $c_{5}$ can be omitted for on-shell processes by a suitable field redefinition and it will therefore be dropped. Using

$$
\Delta=u \lambda_{6} u^{\dagger}
$$

we can write $(2.7)$ in the form

$$
\mathcal{L}_{W_{2}}^{8}=c_{2}<\Delta u_{\mu} u^{\mu}>
$$

The coupling $c_{2}$ is related to the constant $G_{8}$ used in [10] through

$$
c_{2}=G_{8} F_{\pi}^{4} .
$$

A complete list of $47 p^{4}$-terms $\sum N_{i} W_{i}^{8}$ has been given before in Ref. [9]. Using the equation of motion for $U$ and the Cayley-Hamilton theorem, one can reduce the number of independent terms to $37[9,27,28]$. These may be rewritten and grouped into two classes: terms which contribute only for neutral external spin-1 fields (photon and $\mathrm{Z}$ boson fields) and others which are non-vanishing only in the presence of charged external spin-1 fields. These latter ones do not contribute to order $G_{F}$. A first step in this direction had already been taken by Esposito-Farèse [28]. However, our list of independent relevant operators given in Table 1 is shorter than the one presented in Ref. [28]. The 27-plet operators can be simplified correspondingly.

The list of the octet operators $W_{i}^{8}$ is given in Table 1 . The couplings $N_{i}$ are the bare ones and they absorb the infinities arising from the loops. We therefore write in analogy to the strong case $[6]$

$$
N_{i}=N_{i}^{r}(\mu)+Z_{i}(4 \pi)^{-2} \mu^{d-4}\left\{\frac{1}{d-4}-\frac{1}{2}\left(\log 4 \pi+\Gamma^{\prime}(1)+1\right)\right\}
$$

where $N_{i}^{r}(\mu)$ are the renormalization scale dependent renormalized coupling constants. The constants $Z_{i}$ are also listed; they are evaluated from the one-loop divergences $[9,27,28]$ and they determine the scale dependence of the $N_{i}$ :

$$
N_{i}^{r}\left(\mu_{2}\right)=N_{i}^{r}\left(\mu_{1}\right)+\frac{Z_{i}}{16 \pi^{2}} \log \frac{\mu_{1}}{\mu_{2}} .
$$


Of course, this scale dependence must cancel in the sum of loop and counterterm contributions to physical amplitudes.

We note the definitions

$$
\begin{aligned}
\hat{\nabla}_{\mu} \Delta & =\partial_{\mu} \Delta+\left[\Gamma_{\mu}+\frac{i}{2} u_{\mu}, \Delta\right]=u D_{\mu} \lambda_{6} u^{\dagger} \\
w_{\mu \nu} & =\nabla_{\mu} u_{\nu}+\nabla_{\nu} u_{\mu} \\
\nabla_{\mu} u_{\nu} & =\partial_{\mu} u_{\nu}+\left[\Gamma_{\mu}, u_{\nu}\right]
\end{aligned}
$$

used in Table 1. Hermiticity requires the $N_{i}$ to be real. The CP-violating Lagrangian is obtained by replacing $\lambda_{6}$ by $\lambda_{7}$ and the $N_{i}$ by different constants $N_{i}^{\prime}$.

The constants $N_{i}$ contribute in particular to the following physical processes:

$N_{1} \ldots N_{4}: K \rightarrow 3 \pi$

$N_{5} \ldots N_{13}: K \rightarrow 2 \pi, 3 \pi$

$N_{14} \ldots N_{18}$ : radiative $K$ decays

$N_{28} \ldots N_{31}$ : radiative $K$ decays (anomalous couplings [29]).

The $N_{19}, \ldots . N_{27} ; N_{32}, \ldots . N_{35}$ can contribute only to processes with external $\mathrm{W}$ bosons (in addition to the non-leptonic weak transition). The contact terms $N_{36}, N_{37}$ are needed for renormalization, but do not contribute to observable physical processes.

\section{Resonances and the weak chiral Lagrangian}

The couplings of resonances to the pseudoscalar mesons were described in Ref. [11] in the strong interaction case; here, this analysis is extended to the weak sector.

Under a chiral $S U(3) \times S U(3)$ transformation, the matrix $u$ defined in Eq. (2.3) transforms as

$$
u(\phi) \rightarrow g_{R} u(\phi) h^{\dagger}(\phi)=h(\phi) u(\phi) g_{L}^{\dagger} .
$$

$u(\phi)$ is an element of the coset space $S U(3)_{L} \otimes S U(3)_{R} / S U(3)_{V}$ and the non-linear transformation [30] $h(\phi)$ is defined by (3.1).

The resonances, taken to be octets $R$ or singlets $R_{1}$, transform according to

$$
\begin{aligned}
R & \rightarrow h(\phi) R h^{\dagger}(\phi) \\
R & =\frac{1}{\sqrt{2}} \sum_{1}^{8} R^{i} \lambda_{i} \\
R_{1} & \rightarrow R_{1} .
\end{aligned}
$$

The fields $u_{\mu}, u_{\mu} u_{\nu}, \chi_{ \pm}, f_{ \pm \mu \nu}$ and $w_{\mu \nu}$ also transform according to (3.2), as can easily be seen from (3.1). In the following we consider meson resonances of the 
type $V\left(1^{--}\right), A\left(1^{++}\right), S\left(0^{++}\right)$and $P\left(0^{-+}\right)$. It is useful to describe $V$ and $A$ by antisymmetric tensor fields $V_{\mu \nu}$ and $A_{\mu \nu}$, respectively [11,31].

\subsection{Weak couplings of the resonances to the pseudoscalar mesons}

It is straightforward to obtain all the desired couplings. One simply replaces in Table 1 the fields $u_{\mu}, u_{\mu} u_{\nu}$, etc. successively by the $V_{\mu \nu}, A_{\mu \nu}, S, P$ and keeps only linear terms in the resonances (higher powers would yield higher orders in the chiral expansion). Note that weak insertions in the kinetic and mass terms of the resonance fields can always be eliminated by field redefinitions. Some care must be used for operators containing an $\epsilon$ tensor.

We then obtain for the $V$ and $A$ fields

$$
\mathcal{L}(R)=\sum_{1}^{10} g_{R}^{i} K_{i}^{R}
$$

where $R=V$ or $A$ and the $K_{i}^{R}$ are given by

$$
\begin{aligned}
& K_{1}^{R}=<\Delta\left\{R_{\mu \nu}, f_{+}^{\mu \nu}\right\}> \\
& K_{2}^{R}=<\Delta\left\{R_{\mu \nu}, f_{-}^{\mu \nu}\right\}> \\
& K_{3}^{R}=i<\Delta\left[R_{\mu \nu}, \tilde{f}_{+}^{\mu \nu}\right]> \\
& K_{4}^{R}=i<\Delta\left[R_{\mu \nu}, \tilde{f}_{-}^{\mu \nu}\right]> \\
& K_{5}^{R}=i<\Delta\left\{R_{\mu \nu},\left[u^{\mu}, u^{\nu}\right]\right\}> \\
& K_{6}^{R}=i<\Delta u_{\mu} R^{\mu \nu} u_{\nu}> \\
& K_{7}^{R}=<\Delta\left[R^{\mu \nu}, \epsilon_{\mu \nu \alpha \beta} u^{\alpha} u^{\beta}\right]> \\
& K_{8}^{R}=<\Delta u_{\alpha}><R_{\mu \nu} \epsilon^{\mu \nu \alpha \beta} u_{\beta}> \\
& K_{9}^{R}=<\hat{\nabla}_{\nu} \Delta\left\{u_{\mu}, R^{\mu \nu}\right\}> \\
& K_{10}^{R}=i \epsilon^{\mu \nu \alpha \beta}<\hat{\nabla}_{\beta} \Delta\left[u_{\alpha}, R_{\mu \nu}\right]>
\end{aligned}
$$

where $\hat{\nabla}_{\mu} \Delta$ was defined in (2.14). Setting $\Delta=1$ corresponds to the strong interaction case, where only $K_{1}^{R}, K_{2}^{R}$ and $K_{5}^{R}$ (or $K_{6}^{R}$ ) survive (and parity must be conserved). For external electromagnetic fields, $K_{9}^{R}$ and $K_{10}^{R}$ do not contribute since $D_{\nu} \lambda_{6}=\partial_{\nu} \lambda_{6}+i e A_{\nu}\left[Q, \lambda_{6}\right]=0$. In deriving (3.4) we used the CPS symmetry $[24,32,9]$. While (3.4) correspond to CP-conserving couplings, the CP violating ones are obtained by substituting $\lambda_{6} \rightarrow \lambda_{7}$.

If the spin-1 fields are $S U(3)$ singlets, the following terms are possible : 


$$
\begin{aligned}
\mathcal{L}\left(R_{1}\right) & =\sum_{i=11}^{14} g_{R}^{i} K_{i}^{R} \\
K_{11}^{R} & =R_{1}^{\mu \nu}<\Delta f_{+\mu \nu}> \\
K_{12}^{R} & =R_{1}^{\mu \nu}<\Delta f_{-\mu \nu}> \\
K_{13}^{R} & =i R_{1}^{\mu \nu}<\Delta\left[u_{\mu}, u_{\nu}\right]> \\
K_{14}^{R} & =R_{1}^{\mu \nu}<\hat{\nabla}_{\nu} \Delta u_{\mu}>.
\end{aligned}
$$

Since these couplings vanish in the case of the strong interactions $(\Delta=1)$, these operators do not contribute to the weak counterterms. This is no longer true in the presence of external singlet fields. Some of the new aspects of singlet fields are treated in the Appendix.

Next, we consider the octet spin-0 fields $S$ and $P$. The operators $u^{\mu} u_{\mu}$ and $\chi_{ \pm}$ are the scalars of $O\left(p^{2}\right)$ which can couple to $S$ and $P$. Consequently, we have the following terms, taking into account the transformation properties under $\mathrm{CP}$ :

$$
\begin{aligned}
\mathcal{L}(S, P) & =\sum_{1}^{8} g_{S}^{i} K_{i}^{S}+\sum_{1}^{5} g_{P}^{i} K_{i}^{P} \\
K_{1}^{S} & =<\Delta\left\{S, \chi_{+}\right\}> \\
K_{2}^{S} & =<\Delta S><\chi_{+}> \\
K_{3}^{S} & =<\Delta\left[S, \chi_{-}\right]> \\
K_{4}^{S} & =<\Delta\left\{S, u^{\mu} u_{\mu}\right\}> \\
K_{5}^{S} & =<\Delta u^{\mu}><u_{\mu} S> \\
K_{6}^{S} & =<\Delta S><u^{\mu} u_{\mu}> \\
K_{7}^{S} & =i<\hat{\nabla}_{\mu} \Delta\left[u^{\mu}, S\right]> \\
K_{8}^{S} & =<u D_{\mu} D^{\mu} \lambda_{6} u^{\dagger} S>
\end{aligned}
$$

Similar to above, the last two terms vanish if only electromagnetic external fields are considered.

As for the octet pseudoscalars, we obtain

$$
\begin{aligned}
& K_{1}^{P}=i<\Delta\left\{P, \chi_{-}\right\}> \\
& K_{2}^{P}=i<\Delta P><\chi_{-}> \\
& K_{3}^{P}=i<\Delta\left[\chi_{+}, P\right]> \\
& K_{4}^{P}=i<\Delta\left[P, u^{\mu} u_{\mu}\right]> \\
& K_{5}^{P}=<\hat{\nabla}_{\mu} \Delta\left\{u^{\mu}, P\right\}>.
\end{aligned}
$$


Finally, the singlet couplings are

$$
\mathcal{L}\left(S_{1}, P_{1}\right)=\tilde{g}_{S}^{1} \tilde{K}_{1}^{S}+\tilde{g}_{S}^{2} \tilde{K}_{2}^{S}+\tilde{g}_{P}^{1} \tilde{K}_{1}^{P}+\tilde{g}_{P}^{2} \tilde{K}_{2}^{P}
$$

with

$$
\begin{aligned}
& \tilde{K}_{1}^{S}=S_{1}<\Delta \chi_{+}> \\
& \tilde{K}_{2}^{S}=S_{1}<\Delta u_{\mu} u^{\mu}> \\
& \tilde{K}_{1}^{P}=i P_{1}<\Delta \chi_{-}> \\
& \tilde{K}_{2}^{P}=P_{1}<\hat{\nabla}_{\mu} \Delta u^{\mu}>.
\end{aligned}
$$

Including the different $V, A$ operators in (3.4) and (3.5), we arrive at 45 couplings of which 10 vanish for external electromagnetic fields.

\subsection{The effective interaction}

We first recall the strong couplings of meson resonances to the pseudoscalars at $O\left(p^{2}\right)[11]$ :

$$
\begin{aligned}
\mathcal{L}_{2}\left[V\left(1^{--}\right)\right]= & \frac{F_{V}}{2 \sqrt{2}}<V_{\mu \nu} f_{+}^{\mu \nu}>+\frac{i G_{V}}{\sqrt{2}}<V_{\mu \nu} u^{\mu} u^{\nu}> \\
\mathcal{L}_{2}\left[A\left(1^{++}\right)\right]= & \frac{F_{A}}{2 \sqrt{2}}<A_{\mu \nu} f_{-}^{\mu \nu}> \\
\mathcal{L}_{2}\left[S\left(0^{++}\right)\right]= & c_{d}<S u_{\mu} u^{\mu}>+c_{m}<S \chi_{+}> \\
& +\tilde{c}_{d} S_{1}<u_{\mu} u^{\mu}>+\tilde{c}_{m} S_{1}<\chi_{+}> \\
\mathcal{L}_{2}\left[P\left(0^{-+}\right)\right]= & i d_{m}<P \chi_{-}>+i \tilde{d}_{m} P_{1}<\chi_{-}>.
\end{aligned}
$$

In the next step, the resonance fields are integrated out using their classical equation of motion. This procedure is standard [11] and will be repeated for completeness in the case of the vectors. Including both the strong and the weak interactions, the couplings of the vectors are written as

$$
\mathcal{L}=<V_{\mu \nu} \mathcal{J}^{\mu \nu}>, \quad \mathcal{J}^{\mu \nu}=\mathcal{J}_{S}^{\mu \nu}+\mathcal{J}_{W}^{\mu \nu}
$$

with the strong currents given by [Eq.(3.11)]

$$
\mathcal{J}_{S}^{\mu \nu}=\frac{F_{V}}{2 \sqrt{2}} f_{+}^{\mu \nu}+\frac{i G_{V}}{2 \sqrt{2}}\left[u_{\mu}, u_{\nu}\right]
$$

and the weak currents by the various terms in (3.4). The equation of motion 


$$
\nabla^{\alpha} \nabla_{\varrho} V^{\varrho \beta}-\nabla^{\beta} \nabla_{\varrho} V^{\varrho \alpha}+M_{V}^{2} V^{\alpha \beta}=-2 \mathcal{J}^{\alpha \beta}
$$

yields to lowest order in the chiral expansion

$$
V^{\alpha \beta}=-\frac{2}{M_{V}^{2}} \mathcal{J}^{\alpha \beta} .
$$

Inserting this expression into the resonance Lagrangian gives the effective fourth-order Lagrangian (to first order in $G_{F}$ ) as

$$
\mathcal{L}_{W_{4}}^{8}(V)=-\frac{2}{M_{V}^{2}}<\mathcal{J}_{S}^{\alpha \beta} \mathcal{J}_{W \alpha \beta}>
$$

With the explicit form of $\mathcal{J}_{W \alpha \beta}$ we obtain from Eq. (3.16) various terms with the structure of $\mathcal{L}_{W_{4}}^{8}$ in Eq. (2.8). The resulting values of the coefficients $N_{i}$ of the operators listed in Table 1 are given in Table 2.

Similarly, the other resonances also contribute to the $N_{i}$. Their contributions are also included in Table 2. Exchange of a singlet pseudoscalar field $P_{1}$ contributes only to $N_{13}$ and $N_{24}$ :

$$
\begin{aligned}
& \frac{c_{2}}{F_{\pi}^{2}} N_{13}^{P_{1}}=-\frac{\tilde{d}_{m} \tilde{g}_{P}^{1}}{M_{P_{1}}^{2}} \\
& \frac{c_{2}}{F_{\pi}^{2}} N_{24}^{P_{1}}=\frac{\tilde{d}_{m} \tilde{g}_{P}^{2}}{M_{P_{1}}^{2}}
\end{aligned}
$$

The resonance contributions in Table 2 obey the following linearly independent relations $\left(N_{i}^{V}\right.$ denotes the vector contribution to $N_{i}$, etc.):

a) vectors $\left(F_{V}=2 \quad G_{V}\right.$ assumed [31])

$$
\begin{gathered}
N_{1}^{V}+N_{2}^{V}+2 N_{3}^{V}=0 \\
2 N_{3}^{V}-3 N_{4}^{V}=0 \\
5 N_{1}^{V}-N_{2}^{V}+3 N_{15}^{V}=0 \\
N_{1}^{V}-2 N_{2}^{V}+3 N_{14}^{V}+3 N_{16}^{V}-6 N_{18}^{V}=0 \\
N_{28}^{V}-N_{30}^{V}=0 \\
N_{16}^{V}-N_{18}^{V}+N_{27}^{V}=0 \\
N_{18}^{V}-N_{37}^{V}=0 \\
2 N_{19}^{V}-N_{25}^{V}=0 \\
N_{29}^{V}-N_{34}^{V}+N_{35}^{V}=0
\end{gathered}
$$


b) axial vectors

$$
\begin{aligned}
N_{29}^{A}+N_{34}^{A} & =0 \\
N_{18}^{A}+2 N_{27}^{A}+N_{37}^{A} & =0
\end{aligned}
$$

c) octet scalars $\left(r=c_{m} / c_{d}\right)$

$$
\begin{aligned}
& r N_{2}^{S}+r N_{4}^{S}-N_{6}^{S}=0 \\
& r N_{9}^{S}+N_{12}^{S}=0 \\
& r\left(2 N_{1}^{S}+5 N_{2}^{S}\right)-6 N_{7}^{S}-\frac{2}{r}\left(N_{10}^{S}+N_{12}^{S}\right)=0 \\
& r\left(N_{1}^{S}-2 N_{2}^{S}\right)+3 N_{8}^{S}-\frac{1}{r}\left(N_{10}^{S}+3 N_{11}^{S}+N_{12}^{S}\right)=0 \\
& r\left(N_{1}^{S}-2 N_{2}^{S}\right)-2 N_{5}^{S}+\frac{1}{r}\left(N_{10}^{S}+N_{12}^{S}\right)=0 \\
& r N_{19}^{S}=r N_{20}^{S}=-r N_{26}^{S}=\frac{1}{2} N_{22}^{S} \\
& N_{12}^{S}=N_{36}^{S}
\end{aligned}
$$

d) singlet scalars $\left(\tilde{r}=\tilde{c}_{m} / \tilde{c}_{d}\right)$

$$
\begin{aligned}
N_{1}^{S_{1}} & =2 N_{2}^{S_{1}}=-2 N_{4}^{S_{1}} \\
\tilde{r} N_{1}^{S_{1}} & =\frac{4}{3} N_{8}^{S_{1}} \\
\tilde{r} N_{7}^{S_{1}} & =N_{11}^{S_{1}}
\end{aligned}
$$

e) octet pseudoscalars

$$
N_{10}^{P}=-N_{36}^{P}, \quad N_{23}^{P}=\frac{3}{2} N_{24}^{P} .
$$

These relations are useful if only one type of resonance dominates the respective operators. Large $-N_{C}$ arguments give $r=\tilde{r}$ [11] which then relates (3.21) and (3.22). 


\section{Models for weak resonance contributions}

The large number of weak couplings between resonance and pseudoscalar fields encountered in Sect. 3 does not allow for predictions which could be tested with available experimental information. In addition, since only symmetry principles were invoked to construct the most general weak resonance Lagrangian linear in the resonance fields to leading order in the derivative expansion, this Lagrangian does not offer any hint about the strong dynamics behind the various couplings. One could attempt to calculate the couplings directly at the fundamental level, using a quark description for the mesons. This has been done for the weak $\pi N \bar{N}$ and $\rho N \bar{N}$ couplings [16] and further work along these lines is possible also for meson couplings.

Here, we consider instead two approaches towards constructing the non-leptonic weak Lagrangian of $O\left(p^{4}\right)$ which both rest on the assumption that the strong chiral Lagrangian or the associated left-chiral current already determine the dominant features of the $\Delta S=1$ effective Lagrangian. It is clear from the outset that such a procedure cannot be expected to yield the complete weak Lagrangian. There will be genuine weak short-distance contributions, for example, which have no equivalent in the strong sector. A prominent example is provided by the electromagnetic penguin contribution to the weak coupling constant $N_{14}$ defined in Sect. 2 [10]. Nevertheless, one could expect that the dominant long-distance contributions to the $N_{i}$ are due to resonance exchange as is the case in the strong sector [11, 12].

Here, an additional comment is in order. Since the strong Lagrangian $\mathcal{L}_{4}$ does not contain terms with an $\epsilon$ tensor, the weak operators $W_{28}^{8}, \ldots \ldots, W_{35}^{8}$ will not be generated in the models discussed below. On the other hand, the chiral anomaly also appears at $O\left(p^{4}\right)$ in the chiral expansion and it has odd intrinsic parity. It has recently been shown $[33,29]$ that the chiral anomaly actually feeds down to the non-leptonic sector and contributes in particular to the physically relevant coupling constants $N_{28}, \ldots, N_{31}$. Since the anomaly is not related to meson resonances, we will not consider those terms in the rest of this paper. However, it should be kept in mind that $N_{28}, \ldots . ., N_{31}$ are a clear case where meson resonance exchange is most certainly not the dominant effect.

The two models in question, the "weak deformation model" (WDM) [19, 27] and the "factorization model" (FM) $[17,18]$, can be formulated without any reference to resonances. However, to the extent that the strong couplings of $O\left(p^{4}\right)$ seem to be saturated by resonance exchange, these models are at the same time models for the resonance contributions to the weak coupling constants $N_{i}$.

Although the two models are based on seemingly unrelated assumptions, they actually turn out to produce the same structure for the weak $O\left(p^{4}\right)$ terms. This a priori unexpected equivalence lends additional support to the underlying assumptions. 
The only difference between the models is that the FM only determines the relative size of the various couplings, whereas the WDM also makes a definite prediction for the overall scale.

We shall first discuss the WDM in some detail. In the second part of this section, the FM will be formulated and its essential equivalence to the WDM will be demonstrated.

\subsection{Weak deformation model (WDM)}

The WDM starts from the trivial observation [19] that the weak octet Lagrangian of lowest order (2.10)

$$
\mathcal{L}_{W_{2}}^{8}=c_{2}<\Delta u_{\mu} u^{\mu}>
$$

can be generated from the corresponding strong Lagrangian

$$
\mathcal{L}_{2}=\frac{F^{2}}{4}<u_{\mu} u^{\mu}+\chi_{+}>
$$

by the replacement

$$
\begin{gathered}
u_{\mu} \rightarrow u_{\mu}+\left\{u_{\mu}, \hat{\Delta}\right\}-\frac{2}{3}<u_{\mu} \hat{\Delta}>1 . \\
\hat{\Delta}=\frac{c_{2}}{F^{2}} \Delta=\frac{c_{2}}{F^{2}} u \lambda_{6} u^{\dagger} .
\end{gathered}
$$

The trace term in Eq. (4.3) is needed for consistency as long as external singlet gauge fields are omitted in the strong effective Lagrangian of Ref. [6].

The previous trivial observation is promoted to a model (WDM) by requiring [19] :

i) that the deformation (4.3) be accompanied by a corresponding deformation of the connection $\Gamma_{\mu}$

$$
\Gamma_{\mu} \rightarrow \Gamma_{\mu}+\frac{i}{2}\left\{u_{\mu}, \hat{\Delta}\right\}-\frac{i}{3}<u_{\mu} \hat{\Delta}>1
$$

in order to leave the right-chiral combination $\Gamma_{\mu}-\frac{i}{2} u_{\mu}$ unchanged as suggested by the left-chiral nature of the weak interactions;

ii) that not only $\mathcal{L}_{W_{2}}^{8}$, but the $\Delta S=1$ effective octet Lagrangian in general can be generated from the corresponding strong Lagrangian by the substitutions (4.3) and (4.4).

Both for the WDM and the FM, there are two possible procedures to arrive at the resonance contributions to $\mathcal{L}_{W_{4}}^{8}$ :

A. Calculate the resonance contributions to the strong Lagrangian $\mathcal{L}_{4}$ and apply a weak deformation (or factorization) afterwards. 
B. Perform the weak deformation (or factorization) to the strong resonance Lagrangian of lowest order first and then eliminate the resonance fields by the equations of motion.

We shall employ the more general method A since it can be implemented without assuming resonance dominance of the strong coupling constants. To the extent that this resonance saturation is satisfied, method B [13] is, however, completely equivalent. The proof of this equivalence to be sketched below for the simplest case of a scalar resonance relies on the fact that both, weak deformation and factorization, are derivations to first order in $G_{F}$.

Consider the coupling

$$
\mathcal{L}_{\text {res }}=S \cdot j
$$

linear in the scalar resonance field $S$. The scalar current $j$ is a functional of the Goldstone fields and transforms generically as

$$
j \stackrel{W D}{\rightarrow} j+\delta j
$$

under a weak deformation where $\delta j=O\left(G_{F}\right)$. The two procedures outlined above then lead to the same weak Lagrangian of $O\left(p^{4}\right)$

$$
\mathcal{L}_{4}^{\Delta S=1}=\frac{j \delta j}{M_{S}^{2}},
$$

if $M_{S}$ is the scalar resonance mass:

A. Scalar resonance exchange yields the strong Lagrangian

$$
\mathcal{L}_{4}=\frac{j^{2}}{2 M_{S}^{2}}
$$

and therefore

$$
\mathcal{L}_{4} \stackrel{W D}{\rightarrow} \frac{(j+\delta j)^{2}}{2 M_{S}^{2}}=\mathcal{L}_{4}+\frac{j \delta j}{M_{S}^{2}}+O\left(G_{F}^{2}\right)
$$

B. Deforming the resonance Lagrangian (4.5) first and eliminating $S$ by the equation of motion

$$
S=\frac{j}{M_{S}^{2}}+\text { higher orders, }
$$

one obtains 


$$
\mathcal{L}_{\text {res }} \stackrel{W D}{\rightarrow} S(j+\delta j)=\mathcal{L}_{\text {res }}+S \delta j \stackrel{\mathrm{EOM}}{=} \mathcal{L}_{\text {res }}+\frac{j \delta j}{M_{S}^{2}}+\text { higher orders }
$$

The strong Lagrangian $\mathcal{L}_{4}[6]$ can be written in the form

$$
\mathcal{L}_{4}=\sum_{i}^{10} L_{i} O_{i}+H_{1} O_{11}+H_{2} O_{12}
$$

with

$$
\begin{gathered}
O_{1}=<u_{\mu} u^{\mu}>^{2}, \quad O_{2}=<u_{\mu} u_{\nu}>^{2}, \quad O_{3}=<u_{\mu} u^{\mu} u_{\nu} u^{\nu}> \\
O_{4}=<u_{\mu} u^{\mu}><\chi_{+}>, \quad O_{5}=<u_{\mu} u^{\mu} \chi_{+}>, \quad O_{6}=<\chi_{+}>^{2} \\
O_{7}=<\chi_{-}>^{2}, \quad O_{8}=\frac{1}{2}<\chi_{+}^{2}+\chi_{-}^{2}>, \quad O_{9}=-i<f_{+}^{\mu \nu} u_{\mu} u_{\nu}> \\
O_{10}=\frac{1}{4}<f_{+\mu \nu}^{2}-f_{-\mu \nu}^{2}>, \quad O_{11}=\frac{1}{2}<f_{+\mu \nu}^{2}+f_{-\mu \nu}^{2}> \\
O_{12}=\frac{1}{4}<\chi_{+}^{2}-\chi_{-}^{2}>.
\end{gathered}
$$

Using the relations $[11,19,31]$

$$
\begin{gathered}
\Gamma^{\mu \nu}=\frac{1}{4}\left[u^{\mu}, u^{\nu}\right]-\frac{i}{2} f_{+}^{\mu \nu} \\
\Gamma_{\mu \nu}=\partial_{\mu} \Gamma_{\nu}-\partial_{\nu} \Gamma_{\mu}+\left[\Gamma_{\mu}, \Gamma_{\nu}\right] \\
\nabla^{\nu} u^{\mu}-\nabla^{\mu} u^{\nu}=f_{-}^{\mu \nu} \\
\nabla_{\mu} A=\partial_{\mu} A+\left[\Gamma_{\mu}, A\right],
\end{gathered}
$$

the Lagrangian $\mathcal{L}_{4}$ can be expressed entirely in terms of the geometrical quantities $u_{\mu}, \Gamma_{\mu}$ and the scalar operators $\chi_{ \pm}$. Only the vielbein and the connection are subject to the deformations (4.3) and (4.4) leading to the following "deformed" operators ${ }^{1}$ in terms of the $W_{i}^{8}$ defined in Sect. 2:

\footnotetext{
1 Except for the scale factor $\frac{c_{2}}{F_{\pi}^{2}}$ to be taken into account in (4.15).
} 


$$
\begin{aligned}
\delta O_{1} & =\frac{16}{3}\left(2 W_{1}^{8}+W_{2}^{8}-W_{4}^{8}\right) \\
\delta O_{2} & =8 W_{3}^{8} \\
\delta O_{3} & =4\left(W_{1}^{8}+W_{2}^{8}-\frac{2}{3} W_{4}^{8}\right) \\
\delta O_{4} & =4 W_{8}^{8} \\
\delta O_{5} & =-W_{5}^{8}+\frac{2}{3} W_{6}^{8}+W_{7}^{8}+2 W_{8}^{8} \\
\delta O_{9} & =\frac{2}{3} W_{1}^{8}+\frac{10}{3} W_{2}^{8}-2 W_{3}^{8}-\frac{4}{3} W_{4}^{8}-W_{14}^{8}+2 W_{15}^{8}-W_{16}^{8}+W_{19}^{8} \\
\delta O_{10} & =-W_{14}^{8}+W_{16}^{8}+W_{18}^{8}-W_{25}^{8}+W_{26}^{8}-\frac{1}{2} W_{27}^{8} \\
\delta O_{11} & =-2 W_{14}^{8}-2 W_{16}^{8}-W_{18}^{8}-2 W_{25}^{8}-2 W_{26}^{8}+W_{37}^{8} \\
\delta O_{6} & =\delta O_{7}=\delta O_{8}=\delta O_{12}=0 .
\end{aligned}
$$

Thus, the weak Lagrangian of $O\left(p^{4}\right)$ takes the form

$$
\mathcal{L}_{W_{4}}^{8(W D M)}=\frac{c_{2}}{F_{\pi}^{2}}\left(\sum_{i=1}^{10} L_{i} \delta O_{i}+H_{1} \delta O_{11}\right)
$$

implying the following relations ${ }^{2}$ for the weak coupling constants $N_{i}$ in terms of the strong low-energy parameters $L_{i}, H_{1}$ :

$$
\begin{aligned}
& N_{1}^{W D M}=\frac{32}{3} L_{1}+4 L_{3}+\frac{2}{3} L_{9} \\
& N_{2}^{W D M}=\frac{16}{3} L_{1}+4 L_{3}+\frac{10}{3} L_{9} \\
& N_{3}^{W D M}=8 L_{2}-2 L_{9} \\
& N_{4}^{W D M}=-\frac{16}{3} L_{1}-\frac{8}{3} L_{3}-\frac{4}{3} L_{9} \\
& N_{5}^{W D M}=-\frac{3}{2} N_{6}^{W D M}=-N_{7}^{W D M}=-L_{5} \\
& N_{8}^{W D M}=4 L_{4}+2 L_{5} \\
& N_{9}^{W D M}=N_{10}^{W D M}=N_{11}^{W D M}=N_{12}^{W D M}=N_{13}^{W D M}=0 \\
& N_{14}^{W D M}=-L_{9}-L_{10}-2 H_{1} \\
& N_{15}^{W D M}=2 L_{9}
\end{aligned}
$$

\footnotetext{
${ }^{2}$ We refrain from writing down relations for the $N_{i}(i \geq 19)$. The constants $N_{28}, \ldots, N_{31}$ are not affected by the weak deformation and the others are in practice not accessible experimentally (cf. Sect. 2).
} 


$$
\begin{aligned}
& N_{16}^{W D M}=-L_{9}+L_{10}-2 H_{1} \\
& N_{17}^{W D M}=0 \\
& N_{18}^{W D M}=L_{10}-H_{1} .
\end{aligned}
$$

The relations for the $N_{i}$ with $i=1, \ldots, 12$ do not show the correct scaling behaviour. Strictly speaking, the WDM (the same is true for the FM) can therefore make sense at best for a specific value of the scale parameter $\mu$. However, this qualification applies equally well to the observed resonance dominance of the $L_{i}$ which only holds for $\mu=O\left(M_{\rho}\right)$. In the same spirit, we may expect the relations (4.16) to be approximately satisfied (if at all) for values of $\mu$ in the resonance region.

The relations (4.16) for $N_{14}, \ldots, N_{18}$ relevant for radiative $K$ decays are scale independent. This can be traced back to the structure of the divergent part of the one-loop functional [27]. However, another problem arises for $N_{14}, N_{16}$ and $N_{18}$ which depend on the constant $H_{1}$ related to charge renormalization. In contrast to the $L_{i}$, the parameter $H_{1}$ is not accessible experimentally and it depends on the regularization procedure employed. The corresponding operator $O_{11}$ is a pure contact term independent of the Goldstone degrees of freedom. Consequently, although resonance $(\mathrm{V}, \mathrm{A})$ exchange contributes to $H_{1}[11], H_{1}$ has no reason to be resonance dominated. In the following, we shall therefore not make any assumption about $H_{1}$.

In the next step, we replace the $L_{i}$ in Eq. (4.16) by their resonance values. We use both the large- $N_{C}$ argument of Ref. [11] to relate the octet and singlet scalar resonance masses and couplings, and the relations of Ref. [31] among the vector and axial-vector resonance parameters, which are well supported by theoretical and phenomenological arguments. Omitting $L_{6}, L_{7}, L_{8}$ (and $H_{2}$ ), which do not appear in the relations (4.16), we have

$$
\begin{gathered}
L_{1}^{R}=\frac{F_{\pi}^{2}}{16 M_{V}^{2}}, \quad L_{2}^{R}=2 L_{1}^{R}, \quad L_{3}^{R}=-6 L_{1}^{R}+\frac{c_{d}^{2}}{2 M_{S}^{2}} \\
L_{4}^{R}=0, \quad L_{5}^{R}=\frac{c_{d} c_{m}}{M_{S}^{2}}, \quad L_{9}^{R}=\frac{F_{\pi}^{2}}{2 M_{V}^{2}}, \quad L_{10}^{R}=-\frac{3 F_{\pi}^{2}}{8 M_{V}^{2}} \\
M_{V} \simeq M_{\rho}, \quad M_{S} \simeq M_{a_{0}} .
\end{gathered}
$$

Inserting these values in the relations (4.16), we finally obtain the predictions of the WDM for the constants $N_{i}$ in resonance approximation:

$$
N_{1}^{W D M}=-\frac{F_{\pi}^{2}}{2 M_{V}^{2}}+\frac{2 c_{d}^{2}}{M_{S}^{2}}
$$




$$
\begin{aligned}
& N_{2}^{W D M}=\frac{F_{\pi}^{2}}{2 M_{V}^{2}}+\frac{2 c_{d}^{2}}{M_{S}^{2}} \\
& N_{3}^{W D M}=0 \\
& N_{4}^{W D M}=-\frac{4 c_{d}^{2}}{3 M_{S}^{2}} \\
& N_{5}^{W D M}=-\frac{3}{2} N_{6}^{W D M}=-N_{7}^{W D M}=-\frac{1}{2} N_{8}^{W D M}=-\frac{c_{d} c_{m}}{M_{S}^{2}} \\
& N_{9}^{W D M}=N_{10}^{W D M}=N_{11}^{W D M}=N_{12}^{W D M}=N_{13}^{W D M}=0 \\
& N_{14}^{W D M}=-\frac{F_{\pi}^{2}}{8 M_{V}^{2}}-2 H_{1} \\
& N_{15}^{W D M}=\frac{F_{\pi}^{2}}{M_{V}^{2}} \\
& N_{16}^{W D M}=-\frac{7 F_{\pi}^{2}}{8 M_{V}^{2}}-2 H_{1} \\
& N_{17}^{W D M}=0 \\
& N_{18}^{W D M}=-\frac{3 F_{\pi}^{2}}{8 M_{V}^{2}}-H_{1} .
\end{aligned}
$$

\subsection{Factorization model (FM)}

At the fundamental level of the standard model, the effective $\Delta S=1$ Hamiltonian [34]

$$
\mathcal{H}_{\text {eff }}^{\Delta S=1}=\frac{G_{F}}{\sqrt{2}} V_{u d} V_{u s}^{*} \sum_{i} C_{i}\left(\mu^{2}\right) Q_{i}+\text { h.c. }
$$

is expressed in terms of four-quark operators $Q_{i}$ and Wilson coefficients $C_{i}\left(\mu^{2}\right)$ depending on the QCD renormalization scale $\mu$. In a recent paper [17], Pich and de Rafael have formulated a general approach how to realize the four-quark operators $Q_{i}$ and consequently $\mathcal{H}_{\text {eff }}^{\Delta S=1}$ at the effective chiral level. One distinguishes between factorizable (leading in $1 / N_{C}$ ) and non-factorizable (non-leading in $1 / N_{C}$ ) contributions.

To leading order in $1 / N_{C}$ and in the QCD coupling constant $\alpha_{s}$, a generic fourquark operator is realized as a product of chiral currents,

$$
\bar{q}_{l L} \gamma^{\mu} q_{k L} \bar{q}_{j L} \gamma_{\mu} q_{i L} \leftrightarrow \frac{\delta S}{\delta l_{l k}^{\mu}} \frac{\delta S}{\delta l_{\mu, j i}},
$$

where $S$ is the effective chiral action and $i, j, k, l$ are flavour indices. Of course, factorization has been applied many times before in non-leptonic weak interactions (see Ref. [17] for a discussion of previous work). However, the approach of Ref. [17] 
allows in principle for a systematic calculation of corrections to factorization (see also [35]), once a model for spontaneous chiral symmetry breaking is implemented [36,4].

For the dominant octet operator

$$
Q_{-}=\bar{s} \gamma^{\mu}\left(1-\gamma_{5}\right) u \bar{u} \gamma_{\mu}\left(1-\gamma_{5}\right) d-\bar{s} \gamma^{\mu}\left(1-\gamma_{5}\right) d \bar{u} \gamma_{\mu}\left(1-\gamma_{5}\right) u
$$

in (4.19), factorization as defined in Eq. (4.20) corresponds to

$$
Q_{-} \leftrightarrow 4<\lambda J^{\mu} J_{\mu}>
$$

with $\lambda=\frac{1}{2}\left(\lambda_{6}-i \lambda_{7}\right)$ and the left-chiral current

$$
J_{\mu}=\frac{\delta S}{\delta l^{\mu}},
$$

if singlet external fields and currents are neglected (see Appendix). To lowest order in CHPT,

$$
J_{\mu}^{(1)}=\frac{\delta S_{2}}{\delta l^{\mu}}=-\frac{i}{2} F^{2} U^{\dagger} D_{\mu} U=-\frac{F^{2}}{2} u^{\dagger} u_{\mu} u
$$

giving rise to the unique chiral structure of $O\left(p^{2}\right)$

$$
Q_{-} \leftrightarrow F^{4}<u \lambda u^{\dagger} u_{\mu} u^{\mu}>.
$$

In fact, to leading $O\left(p^{2}\right)$ all factorizable and non-factorizable contributions must be of this form adding up to $\mathcal{L}_{W_{2}}^{8}$ in Eq. (4.1).

To $O\left(p^{4}\right)$, the $\mathrm{FM}$ for $\mathcal{L}_{W_{4}}^{8}$ is defined by

$$
\begin{gathered}
\mathcal{L}_{W_{4}}^{8(F M)}=4 k_{f} G_{8}<\lambda\left\{J_{\mu}^{(1)}, J^{(3) \mu}\right\}>+ \text { h.c. } \\
J_{\mu}^{(3)}=\frac{\delta S_{4}}{\delta l^{\mu}} \quad, \quad G_{8}=\frac{c_{2}}{F_{\pi}^{4}} .
\end{gathered}
$$

If factorization is a sensible approximation, we expect the scale factor $k_{f}$ to be $O(1)$. The basic factorization relation (4.20) contains no reference to the QCD scale $\mu$. Consequently, the non-leading corrections must provide the necessary dependence on $\mu[17]$ to compensate the scale dependence of the Wilson coefficients $C_{i}\left(\mu^{2}\right)$ in the effective Hamiltonian (4.19). Our inability to calculate those corrections in a modelindependent way requires the fudge factor $k_{f}$ in Eq. (4.26). This should be regarded as a minimal improvement of naive factorization $\left(k_{f}=1\right)$ : there is no guarantee in general that all terms in Eq. (4.26) have the same scale factor. The FM assumes this to be a reasonable approximation with $k_{f}$ left as a free parameter.

We now turn to the proof of our earlier assertion that to $O\left(p^{4}\right)$ the WDM is a special case of the FM with $k_{f}=\frac{1}{2}$. Before doing so, we emphasize the practical 
consequences of this equivalence. To obtain relations for the $N_{i}^{F M}$ in terms of the $L_{i}$ (and $H_{1}$ ), we can just take over the previously derived relations (4.16) and (4.18) for the WDM and multiply the right-hand sides by a common factor $2 k_{f}$. It is those relations with arbitrary $k_{f}$ that we shall use in Sect. 5 for the phenomenological discussion.

Recalling Eq. (4.24) for $J_{\mu}^{(1)}$, we write $\mathcal{L}_{W_{4}}^{8(F M)}$ in $(4.26)$ as

$$
\begin{aligned}
\mathcal{L}_{W_{4}}^{8(F M)} & =-2 k_{f} G_{8} F^{2}<\lambda_{6}\left\{u^{\dagger} u^{\mu} u, J_{\mu}^{(3)}\right\}> \\
& =-2 k_{f} G_{8} F^{2}<u \lambda_{6} u^{\dagger}\left\{u^{\mu}, u J_{\mu}^{(3)} u^{\dagger}\right\}> \\
& =-2 k_{f} G_{8} F^{2}<\left\{\Delta, u^{\mu}\right\} u J_{\mu}^{(3)} u^{\dagger}>.
\end{aligned}
$$

On the other hand, the WDM can be obtained as

$$
\begin{gathered}
S_{4}\left[u_{\mu}, \Gamma_{\mu}, \chi_{ \pm}\right] \stackrel{W D}{\longrightarrow} S_{4}\left[u_{\mu}, \Gamma_{\mu}, \chi_{ \pm}\right]+\int d^{4} x \mathcal{L}_{W 4}^{8(W D M)}+O\left(G_{F}^{2}\right) \\
\mathcal{L}_{W_{4}}^{8(W D M)}=G_{8} F^{2}<\left\{u_{\mu}, \Delta\right\}\left(\frac{\delta S_{4}}{\delta u_{\mu}}+\frac{i}{2} \frac{\delta S_{4}}{\delta \Gamma_{\mu}}\right)>.
\end{gathered}
$$

By verifying that

$$
J_{\mu}^{(3)}=\frac{\delta S_{4}}{\delta l^{\mu}}=-u^{\dagger}\left(\frac{\delta S_{4}}{\delta u^{\mu}}+\frac{i}{2} \frac{\delta S_{4}}{\delta \Gamma^{\mu}}\right) u,
$$

one indeed finds equivalence between the two models for $k_{f}=\frac{1}{2}$. Note that as everywhere in this section we have omitted ${ }^{3}$ singlet external gauge fields and currents.

\section{Phenomenology}

In this section we turn to the numerical analysis. We will consider both the purely pionic decay modes and those with additional photons and leptons in the final state.

\section{1. $K \rightarrow 2 \pi, 3 \pi$}

We will show that the general factorization model fares relatively well for these decay modes. However, the factorization model with vector exchange only [13] does not reproduce the data adequately.

In general, any physical amplitude can be written as

\footnotetext{
${ }^{3}$ See the Appendix for the modifications due to singlet fields.
} 


$$
A=A_{\text {tr }}+A_{\text {loop }}(\mu)+A_{c t}(\mu)
$$

where $A_{t r}, A_{\text {loop }}$ and $A_{c t}$ are, respectively, the leading order term of order $p^{2}$, the loop contribution and the counterterm piece of order $p^{4}$. For the weak coupling constants of $O\left(p^{4}\right)$, the dependence on the renormalization scale $\mu$ is given in Eq. (2.13) and Table 1; the strong counterterms were analyzed in [6]. We note that both weak and strong counterterms enter $A_{c t}[37,20]$. Of course, the $\mu$ dependences of $A_{\text {loop }}$ and $A_{c t}$ must cancel.

Resonances give $\mu$-independent contributions to counterterms. At first sight, this seems unfortunate since the amplitude $A$ in (5.1) then remains $\mu$-dependent. However, we should think of the resonance picture as a description which is only valid near a certain scale, typically $M_{\rho}$ [11]. We expect resonance dominance to be especially useful if the scale dependence of the corresponding loop contribution is weak.

We consider now the octet amplitudes $A_{0}, \alpha_{1}, \beta_{1}, \zeta_{1}$ and $\xi_{1}$ of $K \rightarrow 2 \pi$ and $K \rightarrow 3 \pi$ decays. Neglecting small terms of $O\left(M_{\pi}^{2} / M_{K}^{2}\right)$, one has the following counterterm contributions [20]

$$
\begin{aligned}
A_{0} & =\frac{2}{F_{\pi}^{2} F_{K}} \sqrt{\frac{2}{3}}\left(M_{K}^{2}-M_{\pi}^{2}\right)\left(-\frac{1}{3} M_{K}^{2} K_{1}\right) \\
\alpha_{1} & =\frac{-2}{27 F_{K} F_{\pi}^{3}} M_{K}^{4}\left\{\left(K_{1}-K_{2}\right)+\frac{4}{F_{\pi}^{2}} 3 c_{2}\left(4 L_{1}+4 L_{2}+2 L_{3}\right)\right\} \\
\beta_{1} & =-\frac{1}{9 F_{K} F_{\pi}^{3}} M_{K}^{2} M_{\pi}^{2}\left\{\left(K_{3}-2 K_{1}\right)+\frac{8}{F_{\pi}^{2}} 3 c_{2}\left(-2 L_{1}+L_{2}-L_{3}+12 L_{4}\right)\right\} \\
\zeta_{1} & =-\frac{1}{6 F_{K} F_{\pi}^{3}} M_{\pi}^{4}\left\{K_{2}-\frac{8}{F_{\pi}^{2}} 3 c_{2}\left(2 L_{1}+2 L_{2}+L_{3}\right)\right\} \\
\xi_{1} & =-\frac{1}{6 F_{K} F_{\pi}^{3}} M_{\pi}^{4}\left\{K_{3}-\frac{8}{F_{\pi}^{2}} 3 c_{2}\left(2 L_{1}-L_{2}+L_{3}\right)\right\} .
\end{aligned}
$$

The $K_{i}$ are combinations of the $N_{i}$ introduced in Sect. 2 as follows:

$$
\begin{aligned}
K_{1} & =\frac{9 c_{2}}{F_{\pi}^{2}}\left(-N_{5}+2 N_{7}-2 N_{8}-N_{9}\right) \\
K_{2} & =\frac{3 c_{2}}{F_{\pi}^{2}}\left(N_{1}+N_{2}+2 N_{3}\right) \\
K_{3} & =\frac{3 c_{2}}{F_{\pi}^{2}}\left(N_{1}+N_{2}-N_{3}\right) .
\end{aligned}
$$

The values to be used are the renormalized ones, taken at the scale $\mu$. The fits of Refs. $[38,20]$ yielded the values of the octet amplitudes in Table 3 . We will always use "reduced amplitudes" in units of $c_{2} / F_{\pi}^{2}$ for convenience (second column of Table 3 ). 
Using the values in the first columns, $c_{2}$ and the $K_{i}$ (in units of $10^{-9}$ ) were determined in $[20]$ to be

$$
\begin{gathered}
\frac{c_{2}}{F_{\pi}^{2}}=(0.67 \pm 0.007) 10^{-7} \\
\\
K_{1}\left(\mu=M_{\eta}, M_{\rho}, 1 \mathrm{GeV}\right)=0.0, \quad 4.5, \quad 7.9 \\
K_{2}\left(\mu=M_{\eta}, M_{\rho}, 1 \mathrm{GeV}\right)=8.4, \quad 7.8, \quad 7.3 \\
K_{3}\left(\mu=M_{\eta}, M_{\rho}, 1 \mathrm{GeV}\right)=5.8, \quad 5.2, \quad 4.7 .
\end{gathered}
$$

While the error on $c_{2}$ is quite small, the errors of the $K_{i}$ need further discussion. In [20] the central values of the $L_{i}$ were used; this then implied small errors of $2 \%$ for $K_{2}$ and $10 \%$ for $K_{3}$. However, there are crucial cancellations in the contributions of the strong counterterms, which still have considerable errors at present. Therefore, the realistic uncertainties for $K_{2}, K_{3}$ are about $30 \%$. This fact must be taken into account when trying to assess the merits of the resonance models.

As mentioned and seen from Eq. (5.5), the scale dependence plays an important role. In order to describe these effects, we write any counterterm amplitude as

$$
A_{c t}(\mu)=A\left(\mu_{0}\right)+\Delta A\left(\mu, \mu_{0}\right)
$$

where $\mu_{0}$ is the renormalization scale and $\Delta A\left(\mu, \mu_{0}\right)$ gives the variation of $A$ under a scale change $\mu_{0} \rightarrow \mu$. $A\left(\mu_{0}\right)$ receives contributions from the $K_{i}$ (weak part) and the $L_{i}$ (strong part). $\Delta A\left(\mu, \mu_{0}\right)$ depends only on $c_{2}$, but not on the values of the $K_{i}, L_{i}$ at $\mu_{0}$. In order to exhibit the sources of the scale dependence clearly, we separate weak and strong effects, writing

$$
\Delta A=\kappa\left(\delta A^{W}+\delta A^{S}\right)
$$

where

$$
\kappa=\frac{c_{2}}{F_{\pi}^{2}} \log \left(\frac{\mu}{\mu_{0}}\right)
$$

corresponding to the weak and strong pieces in $A\left(\mu_{0}\right)^{4}$. The $\delta A^{W, S}$ can be determined from Eq. (2.13) and from Ref. [6]. The numerical values are displayed in Table 4. We have separated also the contribution due to $K_{1}$. We see that it varies rather strongly with $\mu$ whereas the $\mu$-dependence is less pronounced for the other terms including the strong ones. An exception is the amplitude $\alpha_{1}$ where, however, the scale dependences of $K_{2}, K_{3}$ and the strong terms cancel to some extent.

With the values of $c_{2}$ in (5.4) and of the $K_{i}$ (Eq. (5.5)) one finds the results for loop and counterterm contributions given in Tables 5 and 6 . The strong contributions due

\footnotetext{
${ }^{4} \mathrm{By}$ this we mean that the scale dependence coming from the weak (strong) counterterms resides in $\delta A^{W}\left(\delta A^{S}\right)$.
} 
to the low-energy constants $L_{i}$ are listed in Table 7. Also included are the resonance dominance values [11]. As already mentioned, the errors on the $L_{i}$ imply very large uncertainties of about $50 \%$ for $\alpha_{1}$ and $\zeta_{1}$. Fortunately, the strong terms are not so important in those cases.

We now come to the resonance model. Without restrictions, we obtain from the general expressions in Table 2

$$
\begin{aligned}
K_{1} & =-\frac{9 c_{d}}{M_{S}^{2}}\left(\frac{7}{3} g_{S}^{1}+2 g_{S}^{2}-g_{S}^{3}\right)+\frac{9 d_{m}}{M_{P}^{2}} g_{P}^{4} \\
& +\frac{9 c_{m}}{M_{S}^{2}}\left(\frac{1}{3} g_{S}^{4}+2 g_{S}^{6}\right)+\frac{18 \tilde{c}_{d}}{M_{S_{1}}^{2}} \tilde{g}_{S}^{1}-\frac{18 \tilde{c}_{m}}{M_{S_{1}}^{2}} \tilde{g}_{S}^{2} \\
K_{2} & =\frac{2 c_{d}}{M_{S}^{2}}\left(g_{S}^{4}+3 g_{S}^{6}\right)+\frac{6 \tilde{c}_{d} \tilde{g}_{S}^{2}}{M_{S_{1}}^{2}} \\
K_{3} & =K_{2}+\frac{9 G_{V}}{\sqrt{2} M_{V}^{2}}\left(-4 g_{V}^{5}+g_{V}^{6}\right) .
\end{aligned}
$$

It is obvious that these expressions allow us to fit the values of the $K_{i}$ in Eq. (5.5). As to the order of magnitude of the couplings, we have from (5.9) and (5.5)

$$
K_{2}-K_{3}=\frac{9 G_{V}}{\sqrt{2} M_{V}^{2}}\left(4 g_{V}^{5}-g_{V}^{6}\right) \simeq 2.6 \cdot 10^{-9}
$$

where the right-hand side is in fact scale independent. Using $G_{V}=53 \mathrm{MeV}, M_{V}=M_{\rho}$ [11], we find

$$
4 g_{V}^{5}-g_{V}^{6} \simeq 0.07 \frac{c_{2}}{F_{\pi}^{2}} G e V .
$$

If we assume that only vector resonances contribute, then only $K_{3} \neq 0$ and $K_{1}=K_{2}=0$. Concerning $\zeta_{1}$, the contributions from weak counterterms (Table 6) are non-zero for all $\mu$ and the strong corrections (Table 7) are relatively small. This suggests that $K_{2} \neq 0$ (see Eq. (5.2)), although the large errors on $\zeta_{1}$ preclude a definite statement. $A_{0}$ clearly requires a non-zero value of $K_{1}$ at $\mu \simeq M_{\rho}$; however, the strong $\mu$ dependence makes a definite statement difficult. Similarly for $\alpha_{1}$ : at $\mu=M_{\rho}$ the weak counterterms contribute a negligible amount, but again the $\mu$-dependence is strong. We conclude that pure vector resonance saturation, which works quite well in the strong sector, is unlikely here even though absolutely firm statements require better measurements of $\zeta_{1}$ or better knowledge of the $L_{i}$.

We now specialize to the factorization model. Instead of (5.9) we have

$$
\begin{aligned}
& K_{1}=\frac{-9 c_{d} c_{m}}{M_{S}^{2}} \frac{c_{2}}{F_{\pi}^{2}} 2 k_{f} \\
& K_{2}=K_{3}=\frac{12 c_{d}^{2}}{M_{S}^{2}} \frac{c_{2}}{F_{\pi}^{2}} 2 k_{f} .
\end{aligned}
$$


The fact that the $K_{i}$ vanish in the factorization model with only vectors was already observed in Ref. [13]. Since $c_{d} c_{m}>0$ [11], (5.12) implies that $K_{1}$ has opposite sign to $K_{2}, K_{3}$ and is negative. From Eq. (5.5), $K_{1}$ is nowhere negative for $\mu \geq M_{\eta}$ in contradiction to (5.12), but the strong $\mu$-dependence makes a definite statement difficult. Secondly, (5.12) implies $K_{2}=K_{3}$. As indicated in (5.10), $K_{2}-K_{3}$ is non-zero (and scale independent) and again in contradiction to (5.12). However, the present large uncertainties of $K_{2}$ of about $30 \%$ cannot yet rule out $K_{2}=K_{3}$. Thirdly, using the values of $c_{d}, c_{m}$ and $M_{S}$ in [11], we get

$$
-K_{1} \simeq K_{2}=K_{3} \simeq\left(0.85 \cdot 10^{-9}\right) 2 k_{f} .
$$

Comparison with (5.5) shows that this would imply a value of $k_{f}$ of about 4, much larger than the preferred values $k_{f} \simeq 1$. Even reducing $K_{2}$ by a factor of 2 (corresponding to its large uncertainties) does not yield a value consistent with $k_{f} \simeq 1$. However, only further experiments will be able to decide whether the factorization model is ruled out.

The results of Isidori and Pugliese [13] correspond to $K_{i}=0$. Apart from the problems with $K_{1}$, vanishing $K_{2}, K_{3}$ exacerbate the difficulties already discussed in connection with Eq. (5.13).

\subsection{Radiative $K$ decays}

The counterterms $W_{14}^{8}, \ldots, W_{18}^{8}$ contribute to radiative $\mathrm{K}$ decays. For ease of notation, we express the coupling constants used in previous work $[37,10,33]$ in terms of the $N_{i}$ of Sect. 2:

$$
\begin{gathered}
w_{1}=-4 N_{14}, \quad w_{2}=2 N_{15}, \quad w_{4}=4 N_{18} \\
w_{1}^{\prime}=-4 N_{16}, \quad w_{2}^{\prime}=2 N_{17} .
\end{gathered}
$$

The radiative $\mathrm{K}$ decay amplitudes of $O\left(p^{4}\right)$ can be divided up in three classes:

- The loop amplitude diverges, requiring a scale-dependent counterterm amplitude. The only fully analyzed processes of this type are $K^{+} \rightarrow \pi^{+} l^{+} l^{-}, K_{S} \rightarrow \pi^{0} l^{+} l^{-}$ [37] and $K_{L} \rightarrow \pi^{0} \pi^{0} l^{+} l^{-}[39]$.

- The loop amplitude is finite, but there is a non-vanishing counterterm amplitude which is then necessarily scale independent. Here, the relevant transitions are $K^{+} \rightarrow \pi^{+} \gamma \gamma[10]$ and $K^{+} \rightarrow \pi^{+} \pi^{0} \gamma[29,33]$.

- Chiral symmetry forbids counterterms for processes like $K_{S} \rightarrow \gamma \gamma$ [40,41], $K_{L} \rightarrow$ $\pi^{0} \gamma \gamma[42,43]$ and $K_{S} \rightarrow \pi^{0} \pi^{0} \gamma \gamma$ [39]. These decays are therefore not sensitive to the weak coupling constants $N_{i}$. 


\subsection{1. $K \rightarrow \pi l^{+} l^{-}$.}

In addition to calculable one-loop contributions, the amplitudes of $O\left(p^{4}\right)$ for the decays $K^{+} \rightarrow \pi^{+} l^{+} l^{-}$and $K_{S} \rightarrow \pi^{0} l^{+} l^{-}$depend on two constants [37]

$$
\begin{aligned}
& w_{+}=\frac{64 \pi^{2}}{3}\left[N_{14}^{r}-N_{15}^{r}+3 L_{9}^{r}\right]+\frac{1}{3} \log \frac{\mu^{2}}{M_{K} M_{\pi}} \\
& w_{S}=\frac{32 \pi^{2}}{3}\left[2 N_{14}^{r}+N_{15}^{r}\right]+\frac{1}{3} \log \frac{\mu^{2}}{M_{K}^{2}},
\end{aligned}
$$

respectively. The scale dependence of the coupling constants is compensated by the explicit logs in the definitions of $w_{+}, w_{S}$, so that these are observable quantities. We shall set $\mu=M_{\rho}$ and assume resonance dominance at this scale.

From the general discussion of Sect. 3 (Table 2 ) one infers that $w_{+}, w_{S}$ are only sensitive to V exchange. Specializing to the FM, we find

$$
\begin{gathered}
w_{+}=\frac{\left(4 \pi F_{\pi}\right)^{2}}{M_{V}^{2}}\left(2-3 k_{f}\right)+\frac{1}{3} \log \frac{M_{\rho}^{2}}{M_{K} M_{\pi}}-\frac{(16 \pi)^{2}}{3} k_{f} H_{1}^{r}\left(M_{\rho}\right)= \\
=2.3\left(2-3 k_{f}\right)+0.73-\frac{(16 \pi)^{2}}{3} k_{f} H_{1}^{r}\left(M_{\rho}\right) \\
w_{S}=\frac{\left(4 \pi F_{\pi}\right)^{2}}{M_{V}^{2}} k_{f}+\frac{1}{3} \log \frac{M_{\rho}^{2}}{M_{K}^{2}}-\frac{(16 \pi)^{2}}{3} k_{f} H_{1}^{r}\left(M_{\rho}\right)= \\
=2.3 k_{f}+0.29-\frac{(16 \pi)^{2}}{3} k_{f} H_{1}^{r}\left(M_{\rho}\right) .
\end{gathered}
$$

The evidence for the importance of genuine weak contributions (those not proportional to the strong counterterms) is much stronger in this case than for the decays $K \rightarrow 2 \pi, 3 \pi$ discussed previously. In the absence of weak counterterms $\left(k_{f}=0\right)$, the theoretical value for $w_{+}$would be in striking disagreement with the recent experimental determination [44]

$$
w_{+}=0.89_{-}^{+} 0.14
$$

As required by experiment, the weak resonance contributions interfere destructively with the strong pole amplitude due to $L_{9}$ for $k_{f}>0$. Unfortunately, the unknown quantity $H_{1}^{r}\left(M_{\rho}\right)$ precludes a quantitative comparison with experiment. However, as suggested by the arguments of Sect. 4 , it is at least phenomenologically acceptable to set $H_{1}^{r}\left(M_{\rho}\right)=0$. With this assumption, $k_{f}$ could be determined rather precisely from the experimental value (5.18). One should keep in mind however that the error for $w_{+}$ is purely experimental and does not include the uncertainties due to $c_{2}$ or higher-order effects. 
For $k_{f}=\frac{1}{2}(\mathrm{WDM})$, we recover the relation

$$
w_{S}=w_{+}+\frac{1}{3} \log \frac{M_{\pi}}{M_{K}}
$$

used in Ref. [37] for the phenomenological analysis. As shown there, the rate for $K_{S} \rightarrow \pi^{0} l^{+} l^{-}$is very sensitive to $w_{S}$ and therefore also to deviations from Eq. (5.19) for $k_{f} \neq \frac{1}{2}$. This is due to a strong destructive interference between loop and counterterm amplitudes if $w_{S}$ is determined from Eqs. (5.18) and (5.19). Of course, with a strong destructive interference at $O\left(p^{4}\right)$ higher-order effects may become especially important. Finally, we emphasize that the difference $w_{S}-w_{+}$is independent of $H_{1}$ for arbitrary values of $k_{f}$. Given the experimental result (5.18), a measurement of $B R\left(K_{S} \rightarrow \pi^{0} e^{+} e^{-}\right)$could pin down $k_{f}$ rather precisely.

\subsection{2. $K^{+} \rightarrow \pi^{+} \gamma \gamma$.}

The amplitude depends on the scale-invariant combination of coupling constants $[10]$

$$
\hat{c}=\frac{128 \pi^{2}}{3}\left[3\left(L_{9}+L_{10}\right)+N_{14}-N_{15}-2 N_{18}\right] .
$$

In general, $\hat{c}$ is sensitive to both $\mathrm{V}$ and $\mathrm{A}$ exchange (cf. Table 2). In the restricted framework of the FM (or WDM), V exchange drops out and one finds

$$
\hat{c}=\frac{\left(4 \pi F_{\pi}\right)^{2}}{M_{V}^{2}}\left(1-2 k_{f}\right)=2.3\left(1-2 k_{f}\right) .
$$

As shown in Ref. [10], the 2-photon spectrum of $K^{+} \rightarrow \pi^{+} \gamma \gamma$ is very sensitive to $\hat{c}$. Once again, we notice destructive interference between the strong $\left(k_{f}=0\right)$ and weak couplings. Note that $\hat{c}$ does not depend on the contact-term coupling $H_{1}$. The forthcoming measurement of this decay should allow for a clean test of the FM in connection with other determinations of $k_{f}$.

\subsection{3. $K^{+} \rightarrow \pi^{+} \pi^{0} \gamma$.}

The so-called electric amplitude for this decay contains the scale-independent constant $[33]$

$$
c_{+}=w_{1}+2 w_{2}-w_{1}^{\prime}+2 w_{2}^{\prime}=4\left(-N_{14}+N_{15}+N_{16}+N_{17}\right),
$$

sensitive to $\mathrm{V}$ and A exchange in general. More specifically, the FM predicts a complete cancellation among the vector contributions. The remaining A exchange gives rise to 


$$
c_{+}=2 k_{f} \frac{F_{\pi}^{2}}{M_{V}^{2}}=0.03 k_{f} .
$$

For reasonable values of $k_{f}, c_{+}$is smaller than the contribution from $N_{14}-N_{15}$ alone which can be calculated ${ }^{5}$ from the measured value (5.18) and the definition (5.15) of $w_{+}$. This is in fact due to the destructive interference between the $\mathrm{V}$ exchange terms.

In view of the very small loop contribution [33], the direct-emission electric amplitude is dominated by the counterterm amplitude proportional to $c_{+}$. A small positive value of $c_{+}$is consistent with the available experimental information [45]. With future higher statistics, it should be possible to extract $c_{+}$from the measurable interference between the direct-emission electric and the Bremsstrahlung amplitudes.

\section{Conclusions}

For the strong interactions, the effective chiral Lagrangian is completely known up to $O\left(p^{4}\right)$. Essentially all the low-energy constants $L_{i}$ have been determined phenomenologically and they can all be understood in terms of meson resonance exchange.

The situation is quite different for the non-leptonic weak interactions. First of all, the number of terms in the octet Lagrangian of $O\left(p^{4}\right)$ is considerably larger than in the strong sector : there are altogether 37 independent operators, of which still 22 are relevant for most $K$ decays of practical interest. We have presented a convenient basis for the octet Lagrangian of $O\left(p^{4}\right)$ emphasizing the relevant terms $(i=1, \ldots, 18 ; 28, \ldots, 31$ in Table 1$)$.

Not only is the number of low-energy constants considerably bigger, but there is also much less experimental information on these parameters. This makes it all the more mandatory to use additional theoretical input. Guided by the resonance saturation in the strong sector, we have undertaken a systematic analysis of the contributions of meson resonances $(\mathrm{V}, \mathrm{A}, \mathrm{S}, \mathrm{P})$ to the weak Lagrangian of $O\left(p^{4}\right)$. The most general case of all possible couplings allowed by the underlying symmetries has very little predictive power. Nevertheless, our analysis establishes unambiguously which type of resonances can contribute to the weak couplings $N_{i}$. Unlike in the case of the strong interactions, there is no a priori information on the resonance couplings (governing the weak decays of resonances). Moreover, there is a discouragingly large number of such couplings (45 in the most general case). Assuming one type of resonance to dominate the $N_{i}$, a number of relations can be written down, but even

\footnotetext{
${ }^{5} N_{14}-N_{15}$ is scale dependent, so it can be measured only at a given scale, say $\mu=M_{\rho}$.
} 
those cannot be tested with available data. Although there are still large uncertainties, pure vector dominance does not seem to account for the data on $K \rightarrow 2 \pi, 3 \pi$.

Additional assumptions are needed to proceed. We have concentrated on two specific models, the factorization model and the weak deformation model. Both start from the basic hypothesis that the strong effective chiral Lagrangian determines also the structure of the corresponding weak Lagrangian. We have shown that the two models are actually equivalent to $O\left(p^{4}\right)$ except for an overall scale factor $k_{f}$ which is fixed in the WDM $\left(k_{f}=\frac{1}{2}\right)$ whereas the FM suggests $k_{f} \simeq 1$. We have confronted these models with the available experimental data. Although the models are quite predictive, depending essentially only on the scale factor $k_{f}$, the results of our analysis are not completely conclusive at the present time.

Turning first to the decays $K \rightarrow 2 \pi, 3 \pi$, we confirm the observation of Isidori and Pugliese [13] that the genuine weak terms of $O\left(p^{4}\right)$ are insensitive to $\mathrm{V}$ and $\mathrm{A}$ exchange. In contrast to their analysis, we find evidence for such weak contributions in the data. Although the uncertainties are large (the dominant local terms of $O\left(p^{4}\right)$ are due to the strong constants $L_{i}$ ), the remaining $\mathrm{S}$ and $\mathrm{P}$ contributions to the weak counterterm amplitudes predicted by the FM do not seem to be sufficient to explain the data.

The situation is more promising for radiative $K$ decays: only relatively few terms contribute at $O\left(p^{4}\right)$ and they are only sensitive to $\mathrm{V}$ and $\mathrm{A}$ exchange, which are known to dominate the strong constants $L_{i}$. The FM (or WDM) predicts a destructive interference between the strong and the weak couplings in many cases, which is definitely seen in $K^{+} \rightarrow \pi^{+} e^{+} e^{-}$[44]. Forthcoming high-precision experiments on radiative $K$ decays will be able to decide whether spin- 1 resonance exchange according to the FM (WDM) gives the dominant contributions to the relevant constants $N_{i}$.

In contrast to the strong sector, we are still far from a complete understanding of the effective chiral Lagrangian for the weak interactions. Although resonance exchange is certainly expected to produce important contributions to the weak coupling constants, direct short-distance effects are known to be non-negligible (e.g., the electromagnetic penguin contribution to $N_{14}$ ) or probably even dominant in some cases (contribution of the chiral anomaly to the $\epsilon$-type couplings $N_{28}, \ldots, N_{31}$ ) . Much more work is necessary, both in theory and experiment, to fully determine the effective chiral Lagrangian of $O\left(p^{4}\right)$ for the non-leptonic weak interactions.

\section{Acknowledgments}

We thank J. Bijnens, C. Bruno, J. Donoghue, R. Funck, A. Pich and E. de Rafael for helpful discussions and suggestions. G.E. is especially grateful to A. Pich and E. de 
Rafael for earlier unpublished work on both the weak deformation and the factorization models where some of the relations of this paper had already been obtained. J.K. would like to thank A. Prieur for warm hospitality. 


\section{Appendix: Singlet external fields}

In this Appendix the models considered in section 4 (FM and WDM) are generalized to the case where singlet external vector and axial-vector fields are included, i.e. $\left.\left\langle v_{\mu}\right\rangle \neq 0,<a_{\mu}\right\rangle \neq 0$. There are then new terms of order $p^{4}$ which have to be added to the Lagrangian $\mathcal{L}_{4}$ of Gasser and Leutwyler in Eq. (4.12) (some of these terms have already been introduced by Donoghue and Wyler [8] in a different context; we repeat part of that analysis here for completeness). In the strong interaction sector, these additional terms (being proportional to $\left\langle u_{\mu}\right\rangle=D_{\mu} \theta$ ) contribute only to Green functions containing the singlet axial-vector current or the winding number density [6]. However, in both the WDM and the FM additional contributions to Green functions containing only the weak Hamiltonian and octet external fields result. As will be shown below, only the coupling constants $N_{4}$ and $N_{6}$ of the weak chiral Lagrangian (2.8) will be affected.

The basic formalism for the inclusion of singlet external fields has been laid out in Ref. [6]. In order to fix the notation, we briefly review this formalism and point out the main differences to the case without singlet external fields. Allowing for singlet external fields, the restriction $\operatorname{det} U=1$ has to be relaxed:

$$
\operatorname{det} U=\exp \{-i \theta(x)\}
$$

$\theta(x)$ is the conjugate variable to the winding number density $\omega=\frac{1}{16 \pi^{2}} G^{\mu \nu} \tilde{G}_{\mu \nu}$. Under chiral transformations

$$
\theta \longrightarrow \theta-2<\beta>, \quad D_{\mu} \theta \longrightarrow D_{\mu} \theta
$$

where $D_{\mu} \theta=\partial_{\mu} \theta+2<a_{\mu}>=<i U^{\dagger} D_{\mu} U>=<u_{\mu}>$ and $\beta$ parametrizes the transformation. The matrix collecting the Goldstone fields in the standard manner, $\tilde{U}(x)$, is related to $U(x)$ by

$$
U(x)=\tilde{U}(x) \exp \left\{-\frac{i}{3} \theta(x)\right\} .
$$

The covariant derivative takes the form

$$
D_{\mu} U=\left\{D_{\mu} \tilde{U}-\frac{i}{3} D_{\mu} \theta \tilde{U}\right\} \exp \left\{-\frac{i}{3} \theta(x)\right\}
$$

where [Eq. (2.4)]

$$
D_{\mu} U=\partial_{\mu} U-i\left(v_{\mu}+a_{\mu}\right) U+i U\left(v_{\mu}-a_{\mu}\right)
$$

and $D_{\mu} \tilde{U}$ involves only the traceless part of $v_{\mu}, a_{\mu}$. 
The objects used in constructing the chiral Lagrangian of order $p^{4}, O_{i}=\left\{u_{\mu}, \Gamma_{\mu}\right.$, $\left.f_{ \pm}^{\mu \nu}, w^{\mu \nu}\right\}$ are no longer traceless:

$$
O_{i}=\tilde{O}_{i}+\frac{1}{3}<O_{1}>1
$$

$\tilde{O}_{i}$ is the traceless part of $O_{i}$. The following relations remain valid when the trace part is included [Eqs. (2.14), (4.13)] :

$$
\begin{aligned}
f_{+}^{\mu \nu} & =2 i \Gamma^{\mu \nu}-\frac{i}{2}\left[u^{\mu}, u^{\nu}\right] \\
f_{-}^{\mu \nu} & =\nabla^{\nu} u^{\mu}-\nabla^{\mu} u^{\nu} \\
\nabla_{\mu} u_{\nu} & =\frac{1}{2} w_{\mu \nu}-\frac{1}{2} f_{-\mu \nu},
\end{aligned}
$$

where $\nabla_{\mu} u_{\nu}=\partial_{\mu} u_{\nu}+\left[\Gamma_{\mu}, u_{\nu}\right]$. The equations of motion for $U$ are modified to

$$
w_{\mu}^{\mu}-\frac{1}{3}<w_{\mu}^{\mu}>1-i\left(\chi_{-}-\frac{1}{3}<\chi_{-}>1\right)=0 .
$$

Finally, the relations following from the Cayley-Hamilton theorem get additional trace terms. The singlet operators are related through

$$
\begin{aligned}
& <u_{\mu} u_{\nu} u^{\mu} u^{\nu}>+2<u_{\mu}^{2} u_{\nu}^{2}>-<u_{\mu} u_{\nu}><u^{\mu} u^{\nu}>-\frac{1}{2}<u_{\mu}^{2}><u_{\nu}^{2}> \\
= & 4<u_{\mu} u_{\nu}^{2}><u^{\mu}>-2<u_{\mu} u_{\nu}><u^{\mu}><u^{\nu}> \\
- & <u_{\mu}^{2}><u_{\nu}><u^{\nu}>+\frac{1}{2}<u_{\mu}><u^{\mu}><u_{\nu}><u^{\nu}>.
\end{aligned}
$$

The relations among the octet operators become $\left(\Delta=u \lambda_{6} u^{\dagger}\right)$ :

$$
\begin{aligned}
2<\Delta u_{\mu}^{2} u_{\nu}^{2}>+<\Delta u_{\mu} u_{\nu}^{2} u^{\mu}>-\frac{3}{2}<\Delta u_{\mu}^{2}><u_{\nu}^{2}>-<\Delta u_{\mu}><u^{\mu} u_{\nu}^{2}> \\
=<\Delta\left\{u_{\mu}^{2}, u_{\nu}\right\}><u^{\nu}>-<\Delta u_{\mu}><u_{\nu}^{2}><u^{\mu}>-\frac{1}{2}<\Delta u_{\mu}^{2}><u_{\nu}><u^{\nu}> \\
\\
\quad<\Delta u_{\nu} u_{\mu}^{2} u^{\nu}>+<\Delta u_{\nu} u_{\mu} u^{\nu} u^{\mu}>+<\Delta u_{\mu}^{2} u_{\nu}^{2}> \\
-<\Delta u_{\mu} u_{\nu}><u^{\mu} u^{\nu}>-\frac{1}{2}<\Delta u_{\mu}^{2}><u_{\nu}^{2}>-<\Delta u_{\nu}><u^{\nu} u_{\mu}^{2}> \\
=<\Delta\left\{u_{\mu}, u_{\nu}^{2}\right\}><u^{\mu}>+<\Delta u_{\nu} u_{\mu} u^{\nu}><u^{\mu}>-\frac{1}{2}<\Delta u_{\mu}><u_{\nu}^{2}><u^{\mu}> \\
-<\Delta u_{\mu}><u^{\mu} u^{\nu}><u_{\nu}>-\frac{1}{2}<\Delta u_{\mu}^{2}><u_{\nu}><u^{\nu}> \\
-<\Delta u_{\mu} u_{\nu}><u^{\mu}><u^{\nu}>+\frac{1}{2}<\Delta u_{\nu}><u^{\nu}><u_{\mu}><u^{\mu}>
\end{aligned}
$$


and

$$
\begin{aligned}
& <\Delta\left\{u_{\mu}^{2}, \chi_{+}\right\}>+<\Delta u_{\mu} \chi_{+} u^{\mu}>-<\Delta u_{\mu}^{2}><\chi_{+}> \\
& -\frac{1}{2}<\Delta \chi_{+}><u_{\mu}^{2}>-<\Delta u_{\mu}><u^{\mu} \chi_{+}> \\
= & <\Delta\left\{u_{\mu}, \chi_{+}\right\}><u^{\mu}>-<\Delta u_{\mu}><\chi_{+}><u^{\mu}>-\frac{1}{2}<\Delta \chi_{+}><u_{\mu}><u^{\mu}>.
\end{aligned}
$$

The generalization of the chiral Lagrangian (4.12) of order $p^{4}$ is then

$$
\begin{aligned}
\overline{\mathcal{L}_{4}}= & \sum_{i=1}^{10} L_{i} O_{i}+H_{1} O_{11}+H_{2} O_{12} \\
& +\lambda_{1}<u_{\mu}><\nabla^{\mu} \chi_{-}>+\lambda_{2}<\tilde{u}_{\mu} \tilde{u}_{\nu}><u^{\mu}><u^{\nu}> \\
& +\lambda_{3}<\tilde{u}_{\mu}^{2}><u_{\nu}><u^{\nu}>+\lambda_{4}<u_{\mu}><u^{\mu}><u_{\nu}><u^{\nu}> \\
& +\lambda_{5}<\tilde{u}_{\mu} \chi_{+}><u^{\mu}>+\lambda_{6}<\chi_{+}><u_{\mu}><u^{\mu}>+\lambda_{7}<\tilde{u}_{\mu} \tilde{u}_{\nu}^{2}><u^{\mu}> \\
& +\lambda_{8}<f_{+\mu \nu}><f_{+}^{\mu \nu}>+\lambda_{9}<f_{-\mu \nu}><f_{-}^{\mu \nu}>+\lambda_{10}<w_{\mu \nu}><w^{\mu \nu}>.
\end{aligned}
$$

The first 12 terms are the familiar ones of Gasser and Leutwyler, but with $\chi$ replaced by $\tilde{\chi} \equiv \chi e^{i \theta / 3}$. The new terms $\sim \lambda_{i}$, the first six of which have also been given in Ref. [8], vanish if singlet external fields are switched off. A possible term $\left\langle w_{\mu \nu} w^{\mu \nu}\right\rangle$ has been eliminated by partial integration and use of Eqs. (A.7, A.8). In the following, we investigate the consequences of the new counterterms $\sim \lambda_{i}$ for the factorization and weak deformation model.

i) Factorization model including singlet external fields (FMS)

Performing the same steps as in section 4.2 , the contributions to $\mathcal{L}_{W_{4}}^{8(F M S)}$ are easily calculated. We are interested only in the coupling constants $N_{i}$ of Eq. (2.8); terms proportional to $\left\langle u_{\mu}\right\rangle$, which are also produced, are neglected. Compared to the case without singlet external fields, we find the modifications

$$
\begin{aligned}
& N_{4}^{F M S}-N_{4}^{F M}=-2 k_{f} \cdot \lambda_{7} \\
& N_{6}^{F M S}-N_{6}^{F M}=-2 k_{f} \cdot \lambda_{5} .
\end{aligned}
$$

The values of all other constants $N_{i}(i \neq 4,6)$ remain unaffected. The result (A.14) is completely general. The special case of resonance exchange dominance is treated below. 
ii) Weak deformation model including singlet external fields (WDMS)

Including singlet external fields, there is an ambiguity in formulating the weak deformation model. The trace term in the deformation $(4.3,4.4)$

$$
\begin{gathered}
u_{\mu} \longrightarrow u_{\mu}+\left\{\hat{\Delta}, u_{\mu}\right\}-\frac{2}{3} \epsilon<\hat{\Delta} u_{\mu}>1 \\
\Gamma_{\mu} \longrightarrow \Gamma_{\mu}+\frac{i}{2}\left\{\hat{\Delta}, u_{\mu}\right\}-\frac{i}{3} \epsilon<\hat{\Delta} u_{\mu}>1
\end{gathered}
$$

was included in order to keep the deformed vielbein traceless [19]. Since $<u_{\mu}>\neq 0$, this consistency requirement is lost. We have included a factor $\epsilon$ in the trace-term of (A.15) in order to parametrize this ambiguity. Splitting the weak deformation for the traceless part and the trace term,

$$
\begin{aligned}
\tilde{u}_{\mu} \longrightarrow \tilde{u}_{\mu}+\left\{\hat{\Delta}, \tilde{u}_{\mu}\right\}-\frac{2}{3}<\hat{\Delta} \tilde{u}_{\mu}>1+\frac{2}{3} \hat{\Delta}<u_{\mu}> \\
<u_{\mu}>\longrightarrow<u_{\mu}>+2(1-\epsilon)<\hat{\Delta} u_{\mu}>
\end{aligned}
$$

(and similarly for $\Gamma_{\mu}$ ), we observe that only the deformation of the trace-term involves $\epsilon$. As a further consistency check, one may verify that the relation (A.9) implied by the Cayley-Hamilton theorem remains valid if a weak deformation is applied. Indeed, inserting (A.16) in (A.9), the terms proportional to $\epsilon$ drop out. The remaining terms yield one of the Cayley-Hamilton relations for the $\Delta S=1$ operators, Eq. (A.11). Hence any value of $\epsilon$ in (A.15) is consistent with the Cayley-Hamilton theorem, as expected.

The trace term of the weak deformation can be fixed if Green functions containing the $\Delta S=1$ Hamiltonian and the singlet axial vector current are considered. The chiral Lagrangian of order $p^{2}[6]$

$$
\mathcal{L}_{2}=\frac{F^{2}}{4}<u_{\mu} u^{\mu}+\chi_{+}>+\frac{H_{0}}{12}<u_{\mu}><u^{\mu}>
$$

is deformed to

$$
\begin{aligned}
\mathcal{L}_{2} \longrightarrow \mathcal{L}_{2} & +\frac{F^{2}}{4}\left(4<\hat{\Delta} u_{\mu} u^{\mu}>-\frac{4}{3} \epsilon<\hat{\Delta} u_{\mu}><u^{\mu}>\right) \\
& +\frac{H_{0}}{12}\left(4(1-\epsilon)<\hat{\Delta} u_{\mu}><u^{\mu}>\right) \\
= & \mathcal{L}_{2}+F^{2}<\hat{\Delta} \tilde{u}_{\mu} \tilde{u}^{\mu}>+\left(\frac{2}{3} F^{2}(1-2 \epsilon)+\frac{H_{0}}{3}(1-\epsilon)\right)<\hat{\Delta} \tilde{u}_{\mu}><u^{\mu}>.
\end{aligned}
$$

$\epsilon$ determines the strength of observables related to $\left\langle\Delta \tilde{u}_{\mu}\right\rangle\left\langle u^{\mu}\right\rangle$.

Turning now to the coupling constants $N_{i}$, we find the following modifications compared to the case without singlet external fields:

$$
\begin{aligned}
& N_{4}^{W D M S}-N_{4}^{W D M}=2(1-\epsilon) \cdot \lambda_{7} \\
& N_{6}^{W D M S}-N_{6}^{W D M}=2(1-\epsilon) \cdot \lambda_{5} .
\end{aligned}
$$


The equivalence of FM (with $k_{f}=\frac{1}{2}$ ) and WDM is maintained only for $\epsilon=3 / 2$.

Finally, we discuss the implications of singlet external fields for resonance exchange. The resonance Lagrangian [11] gets four additional terms

$$
\begin{aligned}
\Delta \mathcal{L}[V, A, P, S]= & \frac{\tilde{F}_{V}}{2 \sqrt{2}} V_{1, \mu \nu}<f_{+}^{\mu \nu}>+\frac{\tilde{F}_{A}}{2 \sqrt{2}} A_{1, \mu \nu}<f_{-}^{\mu \nu}> \\
& +c_{s}<S \tilde{u}_{\mu}><u^{\mu}>+\tilde{c}_{s} S_{1}<u_{\mu}><u^{\mu}>.
\end{aligned}
$$

Integrating out $V_{1}, A_{1}, S, S_{1}$ results in contributions to the $\lambda_{i}$ :

$$
\begin{aligned}
& \lambda_{3}=\frac{c_{s}^{2}}{2 M_{S}^{2}}+\frac{\tilde{c}_{d} \tilde{c}_{s}}{M_{S_{1}}^{2}}, \quad \lambda_{4}=\frac{\tilde{c}_{s}^{2}}{2 M_{S_{1}}^{2}}, \\
& \lambda_{5}=\frac{c_{m} c_{s}}{M_{S}^{2}}, \quad \lambda_{6}=\frac{\tilde{c}_{m} \tilde{c}_{s}}{M_{S_{1}}^{2}} \\
& \lambda_{7}=\frac{c_{d} c_{s}}{M_{S}^{2}}, \quad \lambda_{8}=-\frac{\tilde{F}_{V}^{2}}{8 M_{V_{1}}^{2}}, \quad \lambda_{9}=-\frac{\tilde{F}_{A}^{2}}{8 M_{A_{1}}^{2}} .
\end{aligned}
$$

The difference (A.19) is given by $\lambda_{5}$ and $\lambda_{7}$. Inserting the values from resonance exchange, we obtain

$$
\begin{aligned}
& N_{4}^{F M S}-N_{4}^{F M}=-2 k_{f} \frac{c_{d} c_{s}}{M_{S}^{2}} \\
& N_{6}^{F M S}-N_{6}^{F M}=-2 k_{f} \frac{c_{m} c_{s}}{M_{S}^{2}}
\end{aligned}
$$

and the same expressions for the WDM, but with the replacement $k_{f} \rightarrow(\epsilon-1)$. The resonance parameter $c_{s}$ in Eqs. (A.20, A.22) has not yet been determined, in contrast to other strong resonance-meson couplings. 
Tables and table captions

Table 1. The 37 operators $W_{i}^{8}$ and the corresponding constants $Z_{i}$.

\begin{tabular}{llr}
$\mathrm{i}$ & \multicolumn{1}{c}{$W_{i}^{8}$} & $Z_{i}$ \\
\hline 1 & $<\Delta u_{\mu} u^{\mu} u_{\nu} u^{\nu}>$ & 2 \\
2 & $<\Delta u_{\mu} u_{\nu} u^{\nu} u^{\mu}>$ & $-\frac{1}{2}$ \\
3 & $<\Delta u_{\mu} u_{\nu}><u^{\mu} u^{\nu}>$ & 0 \\
4 & $<\Delta u_{\mu}><u^{\mu} u_{\nu} u^{\nu}>$ & 1 \\
5 & $<\Delta\left\{\chi_{+}, u_{\mu} u^{\mu}\right\}>$ & $\frac{3}{2}$ \\
6 & $<\Delta u_{\mu}><\chi_{+} u^{\mu}>$ & $-\frac{1}{4}$ \\
7 & $<\Delta \chi_{+}><u_{\mu} u^{\mu}>$ & $-\frac{9}{8}$ \\
8 & $<\Delta u_{\mu} u^{\mu}><\chi_{+}>$ & $-\frac{1}{2}$ \\
9 & $<\Delta\left[\chi_{-}, u_{\mu} u^{\mu}\right]>$ & $\frac{3}{4}$ \\
10 & $<\Delta \chi_{+}^{2}>$ & $\frac{2}{3}$ \\
11 & $<\Delta \chi_{+}><\chi_{+}>$ & $-\frac{13}{18}$ \\
12 & $<\Delta \chi_{-}^{2}>$ & $-\frac{5}{12}$ \\
13 & $<\Delta \chi_{-}><\chi_{-}>$ & 0 \\
14 & $i<\Delta\left\{f_{+}^{\mu \nu}, u_{\mu} u_{\nu}\right\}>$ & $\frac{1}{4}$ \\
15 & $i<\Delta u_{\mu}^{\mu \nu} u_{\nu}>$ & $\frac{1}{2}$ \\
16 & $i<\Delta\left\{f_{-}^{\mu \nu}, u_{\mu} u_{\nu}\right\}>$ & $-\frac{1}{4}$ \\
17 & $i<\Delta u_{\mu} f_{-}^{\mu \nu} u_{\nu}>$ & 0 \\
18 & $<\Delta\left(f_{+\mu \nu}^{2}-f_{-\mu \nu}^{2}\right)>$ & $-\frac{1}{8}$ \\
& & \\
\hline & & \\
19 & $<\hat{\nabla}_{\mu} \Delta\left[u^{\mu}, u_{\nu} u^{\nu}\right]>$ & $-\frac{5}{4}$ \\
20 & $<\hat{\nabla}_{\mu} \Delta\left\{w^{\mu \nu}, u_{\nu}\right\}>$ & $\frac{3}{4}$ \\
21 & $i<\hat{\nabla}_{\mu} \Delta\left[\chi_{+}, u^{\mu}\right]>$ & $\frac{5}{6}$ \\
22 & $<\hat{\nabla}_{\mu} \Delta \nabla^{\mu} \chi_{+}>$ & $\frac{5}{6}$ \\
23 & $\left.i<f_{\mu}^{\mu \nu}, u_{\nu}\right\}>$ \\
24 & $\left.\left.\left.<\hat{\nabla}_{\mu} \Delta u_{-}^{\mu}, u^{\mu}\right\}><\chi_{+}^{2}\right\rangle=\left\{f_{+\mu \nu}, f_{-}^{\mu \nu}\right\}\right)>$ & 0 \\
25 & & $\frac{3}{8}$ \\
26 & & 0 \\
27 & & $\frac{1}{2}$ \\
& &
\end{tabular}




\begin{tabular}{llr}
28 & $i \epsilon_{\mu \nu \rho \sigma}<\Delta u^{\mu}><u^{\nu} u^{\rho} u^{\sigma}>$ & 0 \\
29 & $<\Delta\left[\tilde{f}_{+\mu \nu}-\tilde{f}_{-\mu \nu}, u^{\mu} u^{\nu}\right]>$ & 0 \\
30 & $<\Delta u_{\mu}><\tilde{f}_{+}^{\mu \nu} u_{\nu}>$ & 0 \\
31 & $<\Delta u_{\mu}><\tilde{f}_{-}^{\mu \nu} u_{\nu}>$ & 0 \\
& & \\
\hline & $i<\hat{\nabla}_{\mu} \Delta\left[\tilde{f}_{+}^{\mu \nu}, u_{\nu}\right]>$ & 0 \\
32 & $i<\hat{\nabla}_{\mu} \Delta\left[\tilde{f}_{-}^{\mu \nu}, u_{\nu}\right]>$ & 0 \\
33 & $<\Delta\left[\tilde{f}_{+\mu \nu}+\tilde{f}_{-\mu \nu}, u^{\mu} u^{\nu}\right]>$ & 0 \\
34 & $i<\Delta\left[f_{+\mu \nu}, \tilde{f}_{-}^{\mu \nu}\right]>$ & 0 \\
35 & & $-\frac{5}{12}$ \\
& & \\
\hline & &
\end{tabular}


Table 2. Resonance contributions to the coupling constants $\frac{c_{2}}{F_{\pi}^{2}} N_{i}$ of Eq. (2.8). The factor $1 / M_{R}^{2}$ is omitted throughout.

\begin{tabular}{|c|c|c|c|c|c|}
\hline $\mathrm{i}$ & $V$ & $A$ & $S$ & $S_{1}$ & $P$ \\
\hline 1 & $-\frac{\sqrt{2}}{3} G_{V}\left(2 g_{V}^{5}-2 g_{V}^{6}\right)$ & - & $\frac{c_{d}}{9}\left(10 g_{S}^{4}+12 g_{S}^{6}\right)$ & $\frac{4}{3} \tilde{c}_{d} \tilde{g}_{S}^{2}$ & - \\
\hline 2 & $-\frac{\sqrt[3]{2}}{3} G_{V}\left(10 g_{V}^{5}-g_{V}^{6}\right)$ & - & $\frac{c_{d}}{9}\left(-4 g_{S}^{4}+6 g_{S}^{6}\right)$ & $\frac{2}{3} \tilde{c}_{d} \tilde{g}_{S}^{2}$ & - \\
\hline 3 & $\sqrt{2} G_{V}\left(2 g_{V}^{5}-\frac{1}{2} g_{V}^{6}\right)$ & - & - & - & - \\
\hline 4 & $\frac{\sqrt{2}}{3} G_{V}\left(4 g_{V}^{5}-g_{V}^{6}\right)$ & - & $\frac{c_{d}}{9}\left(4 g_{S}^{4}-6 g_{S}^{6}+9 g_{S}^{5}\right.$ & $-\frac{2}{3} \tilde{c}_{d} \tilde{g}_{S}^{2}$ & - \\
\hline 5 & - & - & $c_{d} g_{S}^{1}+c_{m} g_{S}^{4}$ & - & - \\
\hline 6 & - & - & $c_{m} g_{S}^{5}$ & - & - \\
\hline 7 & - & - & $c_{m} g_{S}^{6}-\frac{2}{3} c_{d} g_{S}^{1}$ & $\tilde{c}_{d} \tilde{g}_{S}^{1}$ & - \\
\hline 8 & - & - & $c_{d} g_{S}^{2}-\frac{2}{3} c_{m} g_{S}^{4}$ & $\tilde{c}_{m} \tilde{g}_{S}^{2}$ & - \\
\hline 9 & - & - & $-c_{d} g_{S}^{3}$ & - & $-d_{m} g_{P}^{4}$ \\
\hline 10 & - & - & $c_{m}\left(2 g_{S}^{1}-g_{S}^{3}\right)$ & - & $d_{m} g_{P}^{3}$ \\
\hline 11 & - & - & $c_{m}\left(g_{S}^{2}-\frac{2}{3} g_{S}^{1}\right)$ & $\tilde{c}_{m} \tilde{g}_{S}^{1}$ & - \\
\hline 12 & - & - & $c_{m} g_{S}^{3}$ & - & $-d_{m}\left(2 g_{P}^{1}+g_{P}^{3}\right)$ \\
\hline 13 & - & - & - & - & $d_{m}\left(\frac{2}{3} g_{P}^{1}-g_{P}^{2}\right)$ \\
\hline 14 & $-\sqrt{2}\left(F_{V} g_{V}^{5}+G_{V} g_{V}^{1}\right)$ & - & - & - & - \\
\hline 15 & $-\frac{\sqrt{2}}{2} F_{V} g_{V}^{6}$ & - & - & - & - \\
\hline 16 & $-\sqrt{2} G_{V} g_{V}^{2}$ & $-\sqrt{2} F_{A} g_{A}^{5}$ & - & - & - \\
\hline 17 & - & $-\frac{\sqrt{2}}{2} F_{A} g_{A}^{6}$ & - & - & - \\
\hline 18 & $-\frac{F_{V}}{2 \sqrt{2}}\left(g_{V}^{1}+g_{V}^{2}\right)$ & $\frac{F_{A}}{2 \sqrt{2}}\left(3 g_{A}^{2}-g_{A}^{1}\right)$ & - & - & - \\
\hline 19 & $\frac{G_{V}}{\sqrt{2}} g_{V}^{9}$ & - & $-\frac{1}{2} c_{d} g_{S}^{8}$ & - & - \\
\hline 20 & - & - & $-\frac{1}{2} c_{d} g_{S}^{8}$ & - & - \\
\hline 21 & - & - & $-c_{m} g_{S}^{7}$ & - & - \\
\hline 22 & - & - & $-c_{m} g_{S}^{8}$ & - & - \\
\hline 23 & - & - & - & - & $-d_{m} g_{P}^{5}$ \\
\hline 24 & - & - & - & - & $-\frac{2}{3} d_{m} g_{P}^{5}$ \\
\hline 25 & $\frac{F_{V}}{\sqrt{2}} g_{V}^{9}$ & - & - & - & - \\
\hline 26 & - & $\frac{F_{A}}{\sqrt{2}} g_{A}^{9}$ & $\frac{1}{2} c_{d} g_{S}^{8}$ & - & - \\
\hline 27 & $\frac{F_{V}}{2 \sqrt{2}}\left(g_{V}^{2}-g_{V}^{1}\right)$ & $\frac{F_{A}}{2 \sqrt{2}}\left(g_{A}^{1}-g_{A}^{2}\right)$ & - & - & - \\
\hline 28 & $-\sqrt{2} G_{V} g_{V}^{8}$ & - & - & - & - \\
\hline 29 & $-\frac{F_{V}}{2 \sqrt{2}} g_{V}^{7}+\frac{G_{V}}{\sqrt{2}}\left(g_{V}^{4}-g_{V}^{3}\right)$ & $\frac{F_{A}}{2 \sqrt{2}} g_{A}^{7}$ & - & - & - \\
\hline 30 & $-\frac{F_{V}^{V}}{\sqrt{2}} g_{V}^{8}$ & - & - & - & - \\
\hline 31 & - & $-\frac{F_{A}}{\sqrt{2}} g_{A}^{8}$ & - & - & - \\
\hline 32 & $\frac{F_{V}}{\sqrt{2}} g_{V}^{10}$ & & - & - & - \\
\hline 33 & - & $\frac{F_{A}}{\sqrt{2}} g_{A}^{10}$ & - & - & - \\
\hline 34 & $\frac{F_{V}}{2 \sqrt{2}} g_{V}^{7}-\frac{G_{V}}{\sqrt{2}}\left(g_{V}^{3}+g_{V}^{4}\right)$ & $-\frac{F_{A}}{2 \sqrt{2}} g_{A}^{7}$ & - & - & - \\
\hline 35 & $-\frac{F_{V}^{V}}{\sqrt{2}} g_{V}^{4}$ & $\frac{\bar{F}_{A}}{\sqrt{2}} g_{A}^{3}$ & - & - & - \\
\hline 36 & $x_{0}-$ & - & $c_{m} g_{S}^{3}$ & - & $-d_{m} g_{P}^{3}$ \\
\hline 37 & $-\frac{F_{V}}{2 \sqrt{2}}\left(g_{V}^{1}+g_{V}^{2}\right)$ & $-\frac{F_{A}}{2 \sqrt{2}}\left(g_{A}^{1}+g_{A}^{2}\right)$ & - & - & - \\
\hline
\end{tabular}


Table 3. Values of the octet amplitudes. In the first column, the values fitted from experiment are given $\left(\alpha_{1}, \beta_{1}, \zeta_{1}, \xi_{1}\right.$ in units of $\left.10^{-8}\right)$. In the second column we list the reduced amplitudes (in units of $c_{2} / F_{\pi}^{2}$; the value of $c_{2} / F_{\pi}^{2}$ in Eq. (5.4) is used). The errors do not include the uncertainty in $c_{2}$.

\begin{tabular}{|c|r|r|}
\hline$A_{0}$ & $0.47 \pm 0.0012 \mathrm{keV}$ & $7.00 \mathrm{GeV}$ \\
\hline$\alpha_{1}$ & $91.7 \pm 0.3$ & $13.6 \pm 0.05$ \\
\hline$\beta_{1}$ & $-25.7 \pm 0.3$ & $-3.83 \pm 0.05$ \\
\hline$\zeta_{1}$ & $-0.47 \pm 0.15$ & $-0.07 \pm 0.023$ \\
\hline$\xi_{1}$ & $-1.5 \pm 0.3$ & $-0.225 \pm 0.045$ \\
\hline
\end{tabular}

Table 4. Values of the $\delta A^{W}, \delta A^{S}$ defined in (5.7). In the weak contributions, the effects from $K_{1}$ are shown explicitly. Unlike the formulas (5.2), also the non-dominant pieces are included.

\begin{tabular}{|r|rrr|r|}
\hline amplitude & $K_{1}$ & rest & tot & $\delta A^{W}$ \\
\hline$A_{0}$ & -6.1 & 0.05 & -6.05 & 0 \\
\hline$\alpha_{1}$ & -9.6 & -2.04 & -11.64 & 3.9 \\
\hline$\beta_{1}$ & 2.1 & 0.16 & 2.36 & 0.17 \\
\hline$\zeta_{1}$ & - & 0.017 & 0.017 & -0.051 \\
\hline$\xi_{1}$ & - & 0.017 & 0.017 & 0 \\
\hline
\end{tabular}

Table 5. Reduced values of tree and loop contributions $\left(A_{t r}\right.$ and $\left.A_{\text {loop }}\right)$ at various scales (units as in the second column of Table 3 ).

\begin{tabular}{|c|r|r|r|r|}
\hline & & \multicolumn{3}{|c|}{ loop (real part) } \\
& tree & $\mu=M_{\eta}$ & \multicolumn{1}{|c|}{$\mu=M_{\rho}$} & $\mu=1 \mathrm{GeV}$ \\
\hline$A_{0}$ & 4.9 & 1.79 & 3.83 & 5.42 \\
\hline$\alpha_{1}$ & 7.71 & 2.26 & 4.86 & 6.88 \\
\hline$\beta_{1}$ & -1.72 & -0.72 & -1.57 & -2.21 \\
\hline$\zeta_{1}$ & - & -0.027 & -0.015 & -0.007 \\
\hline$\xi_{1}$ & - & -0.115 & -0.121 & -0.125 \\
\hline
\end{tabular}


Table 6. Numerical contributions from weak counterterms. The contribution from $K_{1}$ is given separately; units as in the second column of Table 3.

\begin{tabular}{|c|c|c|c|c|c|c|}
\hline \multirow[b]{2}{*}{ amplitude } & \multicolumn{2}{|c|}{$\mu=M_{\eta}$} & \multicolumn{2}{|c|}{$\mu=M_{\rho}$} & \multicolumn{2}{|c|}{$\mu=1 \mathrm{GeV}$} \\
\hline & $K_{1}$ & others & $K_{1}$ & others & $K_{1}$ & others \\
\hline$A_{0}$ & 0 & 0 & -2.06 & 0.02 & -3.66 & 0.03 \\
\hline$\alpha_{1}$ & 0 & 4.68 & -3.25 & 4.0 & -5.76 & 3.46 \\
\hline$\beta_{1}$ & 0 & -0.56 & -0.71 & -0.5 & 1.26 & -0.54 \\
\hline$\zeta_{1}$ & & -0.075 & & -0.069 & & -0.064 \\
\hline$\xi_{1}$ & & -0.052 & & -0.046 & & -0.042 \\
\hline
\end{tabular}

Table 7. Values of the strong counterterm contributions to the various amplitudes. The first three columns are for the values of the counterterms determined in [6] (for renormalization scales $M_{\eta}, M_{\rho}$ and $1 \mathrm{GeV}$ ), the last two for the resonance dominance values [11]; units as in the second column of Table 3.

\begin{tabular}{|r|rrr|r|r|}
\hline amplitude & $M_{\eta}$ & $M_{\rho}$ & $1 \mathrm{GeV}$ & \multicolumn{1}{l|}{ Res. } & vector only \\
\hline$\alpha_{1}$ & -1.01 & 0.32 & 1.34 & -0.96 & 0 \\
\hline$\beta_{1}$ & -0.81 & -0.74 & -0.69 & -0.56 & -0.56 \\
\hline$\zeta_{1}$ & 0.011 & -0.006 & -0.02 & 0.009 & 0 \\
\hline$\xi_{1}$ & -0.062 & -0.062 & -0.062 & -0.043 & -0.052 \\
\hline
\end{tabular}




\section{References}

[1] Proc. of the Ringberg Workshop on Hadronic Matrix Elements and Weak Decays, Ringberg Castle, Germany, April 1988, Eds. A.J. Buras, J.-M. Gérard and W. Huber, Nucl. Phys. B(Proc. Suppl.)7A (1989) .

[2] Proc. of the Workshop on Effective Field Theories of the Standard Model, Dobogókö, Hungary, Aug. 1991, Ed. U.-G. Meißner, World Scient. Publ. Co. (Singapore, 1992).

[3] S. Weinberg, Physica 96A (1979) 327;

H. Georgi, "Weak Interactions and Modern Particle Theory", Benjamin/Cummings (Menlo Park, 1984);

J.F. Donoghue, E. Golowich and B.R. Holstein, "Dynamics of the Standard Model", Cambridge Univ. Press (Cambridge, 1992).

[4] J. Bijnens, C. Bruno and E. de Rafael, "Nambu-Jona-Lasinio like models and the low energy effective action of QCD", preprint CERN-TH.6521/92.

[5] J. Gasser and H. Leutwyler, Ann. Phys. 158 (1984) 142.

[6] J. Gasser and H. Leutwyler, Nucl. Phys. B250 (1985) 465.

[7] J.F. Donoghue and H. Leutwyler, Z. Phys. C52 (1991) 343.

[8] J.F. Donoghue and D. Wyler, Phys. Rev. D45 (1992) 892.

[9] J. Kambor, J. Missimer and D. Wyler, Nucl. Phys. B346 (1990) 17.

[10] G. Ecker, A. Pich and E. de Rafael, Nucl. Phys. B303 (1988) 665.

[11] G. Ecker, J. Gasser, A. Pich and E. de Rafael, Nucl. Phys. B321 (1989) 311.

[12] J.F. Donoghue, C. Ramirez and G. Valencia, Phys. Rev. D39 (1989) 1947.

[13] G. Isidori and A. Pugliese, "Chiral weak Lagrangian for vector mesons and $K \rightarrow 3 \pi$ decay amplitudes", Univ. di Roma "La Sapienza" preprint n. 849, Dec. 1991.

[14] See, e.g., R.J. Blin-Stoyle, "Fundamental Interactions and the Nucleus", North Holland (Amsterdam, 1973).

[15] For a recent overview, see W.T.H. Van Oers, "Symmetries and the NN-interaction", Univ. of Manitoba preprint 1992.

[16] B. Desplanques, J.F. Donoghue and B.R. Holstein, Ann. Phys. (N.Y.) 124 (1980) 449;

B.R. Holstein, "Weak Interactions in Nuclei", Princeton Univ. Press (1989).

[17] A. Pich and E. de Rafael, Nucl. Phys. B358 (1991) 311.

[18] H.-Y. Cheng, Phys. Rev. D42 (1990) 72.

[19] G. Ecker, A. Pich and E. de Rafael, Phys. Lett. B237 (1990) 481. 
[20] J. Kambor, J. Missimer and D. Wyler, Phys. Lett. B261 (1991) 496.

[21] J. Kambor, J.F. Donoghue, B.R. Holstein, J. Missimer and D. Wyler, Phys. Rev. Lett. 68 (1992) 1818.

$[22]$ G. Ecker, "Chiral realization of the non-leptonic weak interaction", in Proc. of the 24th Int. Symposium on the Theory of Elementary Particles, Gosen, Germany, Oct. 1990, Ed. G. Weigt (Zeuthen, 1991).

[23] J.A. Cronin, Phys. Rev. 161 (1967) 1483.

[24] C. Bernard, T. Draper, A. Soni, H. Politzer and M. Wise, Phys. Rev. D32 (1985) 2343.

[25] R.J. Crewther, Nucl. Phys. B264 (1986) 277.

[26] M. Leurer, Phys. Lett. B201 (1988) 128.

[27] G. Ecker, "Geometrical aspects of the non-leptonic weak interactions of mesons", in Proc. of the IX. International Conference on the Problems of Quantum Field Theory, Dubna, USSR, April 1990, Ed. M.K. Volkov (Dubna, 1990).

[28] G. Esposito-Farèse, Z. Phys. C50 (1991) 255.

[29] J. Bijnens, G. Ecker and A. Pich, "The chiral anomaly in non-leptonic weak interactions", preprint CERN-TH.6444/92, to appear in Phys. Lett. B.

[30] S. Coleman, J. Wess and B. Zumino, Phys. Rev. 177 (1969) 2239;

C. Callan, S. Coleman, J. Wess and B. Zumino, Phys. Rev. 177 (1969) 2247.

[31] G. Ecker, J. Gasser, H. Leutwyler, A. Pich and E. de Rafael, Phys. Lett. B223 (1989) 425.

[32] S.P. Rosen, Phys. Rev. 137 (1965) B431.

[33] G. Ecker, H. Neufeld and A. Pich, Phys. Lett. B278 (1992) 337.

[34] F.J. Gilman and M.B. Wise, Phys. Rev. D20 (1979) 1216.

[35] C. Bruno, J. Prades and E. de Rafael, in preparation.

[36] D. Espriu, E. de Rafael and J. Taron, Nucl. Phys. B345 (1990) 22; Err. ibid. B355 (1991) 278.

[37] G. Ecker, A. Pich and E. de Rafael, Nucl. Phys. B291 (1987) 692.

[38] T.J. Devlin and J.O. Dickey, Rev. Mod. Phys. 51 (1979) 237.

[39] R. Funck and J. Kambor, The decays $K_{L, S} \rightarrow \pi^{0} \pi^{0} \gamma \gamma$ and $K_{L} \rightarrow \pi^{0} \pi^{0} l^{+} l^{-}$in the effective chiral lagrangian approach, preprint TUM-T31-24/92.

[40] G. D’Ambrosio and D. Espriu, Phys. Lett. B175 (1986) 237. 
[41] J.L. Goity, Z. Phys. C34 (1987) 341.

[42] G. Ecker, A. Pich and E. de Rafael, Phys. Lett. B189 (1987) 363.

[43] L. Cappiello and G. D'Ambrosio, Nuovo Cim. 99A (1988) 153.

[44] C. Alliegro et al., Phys. Rev. Lett. 68 (1992) 278.

[45] R.J. Abrams et al., Phys. Rev. Lett. 29 (1972) 1118;

V.N. Bolotov et al., Sov. J. Nucl. Phys. 45 (1987) 1023. 